\title{
AN EXPERIMENTAL STUDY OF THE CONTRIBUTION OF NUCLEAR FISSION TO THE SIGNAL OF URANIUM HADRON CALORIMETERS
}

\author{
Claude Leroy ${ }^{1}$, Yves Sirois ${ }^{1}$ and Richard Wigmans ${ }^{2}$ \\ CERN, Geneva, Switzerland
}

\begin{abstract}
We determined the number of fissions that occur in the development of a hadron shower in a ${ }^{238} \mathrm{U}$ calorimeter, using a method based on the analysis of induced radioactivity. Measurements were done at $300 \mathrm{GeV}\left(\pi^{-}\right)$, and at $591 \mathrm{MeV}$ (protons). The number of fissions turns out to be much smaller than usually assumed ( $\sim 10$ fissions per $\mathrm{GeV}$ ), and is very sensitive to the calorimeter configuration. For example, in massive ${ }^{238} \mathrm{U}$ the number of neutron-induced fissions is $25 \%$ larger than in a fine-sampling uranium/scintillator device. The results for calorimeters with a high- $Z$ readout are similar to the massive $U$ case. We also determined the total neutron production in the proton beam. It turns out that the insertion of low-Z readout layers has a great impact on the neutron flux. The neutron flux in lead is about $40 \%$ of that in uranium, but the neutrons are on an average faster. We measured that $4.1 \pm 0.4$ fissions per $\mathrm{GeV}$ are on an average induced in the shower development of an incoming $300 \mathrm{GeV} \pi^{-}$. An attempt is made to explain these data.

The consequences for calorimetric measurements of the hadron energy are discussed.
\end{abstract}

(Submitted to Nuclear Instruments and Methods in Physics Research)

1 McGill University, Montreal, Canada.

2 On leave of absence from NIKHEF, Amsterdam, the Netherlands. 


\section{INTRODUCTION}

The use of calorimeter or total-absorption techniques is rapidly gaining importance in detectors for high-energy physics experiments. In the next generation of colliding-beam experiments (at SLC, HERA, Tevatron) large hadron calorimeters will be the key instruments. In order to be able to optimally design these detectors, it is of vital interest to understand the factors that limit their performance in as much detail as possible. Amongst the various options, there is one that seems particularly attractive: the choice of uranium as absorber material. Apart from the fact that this makes it possible to construct very compact devices, and that the natural radioactivity provides a stable calibration signal for free, the main advantage of uranium is the possibility of considerably improving the achievable energy resolution for hadron showers. This was first demonstrated experimentally by the R807 group at CERN [1]. Recently, their uranium/scintillator detector was reconstructed for the HELIOS experiments and tested at the Super Proton Synchrotron (SPS). The energy resolution for pions was found to be close to $35 \% \mathrm{E}^{-1 / 2}$ up to $200 \mathrm{GeV}$ [2]. On the other hand, tests by various other groups, mainly with liquid-argon readout, did not reproduce these results. Energy resolutions were found to be larger than $50 \% \mathrm{E}^{-1 / 2}$ and did not scale with $\mathrm{E}^{-1 / 2}$ [3]. This indicates that details of the design, i.e. sampling width, or the properties of the active medium may be of crucial importance for achieving the optimal performance. It has been pointed out many times [4-8] that, to obtain the best energy resolution possible, the detector should have equal response to electromagnetic and purely hadronic showers of the same energy. In this way, the contribution to the energy resolution of the large event-to-event fluctuations in the fraction of the energy used for $\pi^{0}$ production in realistic hadron showers may be limited considerably. Values of e/ $\pi$ close to 1.0 have been reported for uranium calorimeters $[2,8,9]$, while for low- $Z$ absorbers one usually finds values $1.3-1.5[8]$.

It is usually assumed that amplification of the hadronic signal due to the contribution of nuclear fission is at least partly responsible for this. It has, however, also become more and more clear that reduction of the response to electromagnetic showers with respect to minimum ionizing particles, as observed experimentally in calorimeters with high- $Z$ absorber, plays an important role as well. The interplay between these phenomena and the influence of the calorimeter parameters on the achievable energy resolution are the subject of a separate article [10]. In this paper, we will present new experimental information that allows the estimation of the contribution of nuclear fission for a particular calorimeter design and illustrates the importance of the choice of the calorimeter parameters. In section 2, the experimental methods and the ideas on which they are based will be described. The results of the measurements are presented in section 3, and discussed in section 4.

\section{EXPERIMENTAL METHODS}

\subsection{The basic idea}

If one wants to estimate how much the uranium calorimeter signals from hadron showers are affected by nuclear fission, one needs to know how many fission processes occur per unit of deposited energy in the block of matter in which the shower develops. As far as we know, this has never been measured directly. What has been measured concerns the flux of neutrons escaping from a uranium target hit by fast particles [11-13].

Extracting the information needed for our purpose from such data is far from trivial. Fabjan [8] assumes that $\mathbf{4 0}$ fissions are induced per $\mathrm{GeV}$ of energy deposited, and that these account for a $30-40 \%$ amplification of the hadronic signal. Monte Carlo simulations claim numbers around 25 fissions per GeV [14].

Our measurements of this crucial number are based on the following idea. Suppose we shoot a high-energy particle into a block of matter that is sufficiently large to fully contain the shower. While the shower develops, numerous nuclear reactions will take place. If the block contains uranium, a certain fraction of such reactions will cause fission of uranium nuclei. The reaction products, and in particular the fission products, will stay inside the block. Therefore, if one is able to count the number of fission products afterwards, one can tell immediately how many fissions have been induced by the particle sent into the block. This counting is, in principle, simple if the reaction products are radioactive. They can be easily identified with high-resolution $\gamma$-ray spectroscopy. In order to limit the effects of absorption of the $\gamma$-rays, the block should be made of thin plates, which can be measured one by one. In this way one can not only measure the absolute number of fissions 
induced, but also the intensity distribution inside the block. The penetrating hadron has left its 'fingerprints' in the form of a profile of induced radioactivity that is frozen inside the block, and that may yield information on the distribution of the particles that caused the nuclear reactions. We will show examples of this.

The block of matter may essentially be anything that one wants to study. So far, we have used the following configurations:

a) blocks made of $3 \mathrm{~mm}$ thick depleted ${ }^{238} \mathrm{U}$ plates;

b) uranium/scintillator configurations $\left[3 \mathrm{~mm}{ }^{238} \mathrm{U}\right.$ alternated with $2.5 \mathrm{~mm}$ acrylic scintillator in a $(1: 1)$ or $(3: 1)$ ratio];

c) uranium/iron, to simulate liquid-argon readout $\left(3 \mathrm{~mm}{ }^{238} \mathrm{U}\right.$ alternated with $\left.0.5 \mathrm{~mm} \mathrm{Fe}\right)$;

d) Lead/uranium ( $5 \mathrm{~cm} \mathrm{~Pb}, 3 \mathrm{~mm}{ }^{238} \mathrm{U}$ ), where the uranium serves to determine the number of neutrons generated in the lead;

e) a block consisting of $5 \mathrm{~mm}$ thick copper plates;

f) a mixed uranium/copper/scintillator configuration.

The lateral dimensions of the plates were $20 \times 120 \mathrm{~cm}^{2}$. These stacks were exposed to either $300 \mathrm{GeV} \pi^{-}$particles from the CERN SPS at a rate of $\sim 5 \times 10^{8}$ per burst, or to protons from the CERN Synchro-cyclotron (SC) with a kinetic energy of $591 \mathrm{MeV}$, at a rate of $\sim 10^{11}$ per second. In the latter case, the stacks were made 3.5-4 nuclear interaction lengths $(\sim 40 \mathrm{~cm}$ of uranium) deep. Less than $1 \%$ of the reaction products are formed beyond this depth. For the high-energy exposure, we used 7.3 interaction lengths $\left(\lambda_{\mathrm{INT}}\right)$ giving about $2 \%$ of rear leakage on an average, as measured with the HELIOS uranium/scintillator calorimeter installed behind the exposed target.

The exposure time was short compared to the typical decay periods (several days) of the nuclides analysed afterwards, so that corrections for decay during the exposure are negligible. Typically, we accumulated $\sim 5 \times 10^{11}$ incident pions and $\sim 5 \times 10^{13}$ incident protons. The number of incident particles was checked with thin aluminium foils placed upstream in the beam. The beam particles cause spallation reactions in $\mathrm{Al}$ for which the cross-section is accurately known. The yield of such reactions allowed the determination of the number of beam particles with an accuracy of about $5 \%$.

Measurements usually started about 3-4 days after the end of the exposure, since the radioactivity had to decrease to an acceptable level before the stack could be taken apart.

The $\gamma$-ray measurements were performed with a $45 \mathrm{~cm}^{3}$ high-purity $\mathrm{Ge}$ crystal with a resolution of $2.1 \mathrm{keV}$ at $1.3 \mathrm{MeV}$. The plates were placed at a distance of $14.4 \mathrm{~cm}$ from the surface of the $\mathrm{Ge}$ counter, aligned in such a way that the centre of exposure lay on the crystal axis (see fig. 1). Since we were only interested in the region of the plate where induced radioactivity is to be found, and in order to limit the background of uranium decay and variations in detector efficiency due to geometry, the region of the plates observed by the detector was chosen to be $20 \times 20 \mathrm{~cm}^{2}$, the rest of the plate being shielded by lead walls around the detector. Corrections for the radioactive products eventually produced outside this region were obtained from separate measurements on the lateral distributions. In this way, the integral radioactivity induced in the plate was recorded. Combining the results of such measurements for all measured plates gives the longitudinal profile of the induced radioactivity. Integration over this profile allowed the extraction of the information needed to determine the number of fissions per GeV. An example of such a longitudinal profile is given in fig. 2 .

Gamma-rays were attributed to the decay of certain radio-isotopes on the basis of their decay period and their energy, measured with a precision of $<0.5 \mathrm{keV}$, cross-checking of course the relative intensities if more than one $\gamma$-ray should be visible according to the well-known decay schemes [15]. Table 1 lists the nuclides that could be unambiguously identified in this way, together with the energies and relative intensities of the $\gamma$-rays that were used for the following analysis. Most of these nuclei are uranium fission products; ${ }^{239} \mathrm{~Np}$ results from slow neutron capture by ${ }^{238} \mathrm{U}$, yielding ${ }^{239} \mathrm{U}$ which decays with a period of $23.5 \mathrm{~min}$ to ${ }^{239} \mathrm{~Np}$, and ${ }^{237} \mathrm{U}$ can be produced in various ways by simple reactions such as $(n, 2 n),(\gamma, n),(\pi, n)$ or $(p, d)$ off ${ }^{238} U$. The list of fission products that we observed is, of course, determined by the length of the period in between the end of the irradiation and the start of the measurements, and the decay periods of these nuclides. Once we performed measurements only a few hours after the end of the irradiation and then the $\gamma$-ray spectra were completely dominated by nuclides such as ${ }^{91} \mathrm{Sr}\left(\mathrm{T}_{1 / 2}=9.5 \mathrm{~h}\right),{ }^{112} \mathrm{Ag}(3.1 \mathrm{~h})$ and ${ }^{135} \mathrm{I}(6.6 \mathrm{~h})$. These half-lives are, however, too short to allow for a systematic study, which requires measurements 
of a considerable number of plates, and usually takes a couple of days. For that reason we preferred to work with an increased dose and to do the analysis on the long-lived activities.

Apart from the mentioned $\gamma$-rays, the spectra contained also some clear lines from the natural ${ }^{238} \mathrm{U} \alpha$-decay chain, in particular the $1001 \mathrm{keV} \gamma$ from ${ }^{234 \mathrm{~m}} \mathrm{~Pa}$ decay. The intensity of this line was used to correct for variations in the uranium-plate thickness. These corrections were small, the $\sigma_{\mathrm{rms}}$ of the thickness distribution amounted to $\sim 3 \%$.

Once the origin of the observed $\gamma$-ray was known, the measured intensities could be easily corrected for the decay between the end of the irradiation and the measurement performed at time $t$. The relation between these intensities $I_{0}$ and the number of nuclei produced during the irradiation $\mathrm{N}_{0}$ is given by the following formula:

where

$$
\mathrm{N}_{0}=\mathrm{I}_{0} / \epsilon_{\gamma} \mathrm{f}_{\gamma} \lambda \mathrm{f}_{\gamma}^{\mathrm{u}} \mathrm{f}_{\gamma}^{\text {wood }} \text { and } \mathrm{I}_{0}=\mathrm{I}(\mathrm{t}) / \mathrm{e}^{-\lambda \mathrm{t}}
$$

$\epsilon_{\gamma}=$ the absolute detector efficiency for photoelectric effect caused by $\gamma$ 's of the energy concerned;

$\mathrm{f}_{\gamma}=$ the fraction of the nuclei that decay with emission of the $\gamma$-ray concerned;

$\lambda=$ the decay period of the radioactive nuclide;

$\mathrm{f}_{\gamma}^{\mathrm{u}}=$ the fraction of $\gamma$ 's that leave the uranium plate undisturbed, i.e. without Compton scattering, etc., in the plate itself;

$\mathrm{f}_{\gamma}^{\text {wood }}=$ the fraction of $\gamma$ 's that traverse a wooden plate, placed in between the uranium and the detector for support reasons, undisturbed.

The values of $f_{\gamma}$ and $\lambda$ are listed in table 1 . Their precision is $\leq 1 \%$. The values of $f_{\gamma}^{u}$ and $f_{\gamma}^{\text {wood }}$ are strongly dependent on the energy of the $\gamma$-ray. We calculated them using the mass-attenuation coefficients given in ref. [16], assuming that the radioactivity is homogeneously distributed in depth over one plate. The resulting curves are given in fig. 3 . They were checked by comparing the relative intensities of different $\gamma$-rays emitted by one nuclide obtained with these curves, to the values given in the literature. In doing so for ${ }^{132} \mathrm{Te}$, which has dominating lines over a broad energy range $(0.2-2 \mathrm{MeV})$, we found that the results are correct within experimental errors $(<5 \%)$.

The absolute efficiency $\epsilon$ as a function of energy was determined with a set of precisely calibrated point sources placed at the same distance from the detector as the uranium plates. The activity in the plates, however, extends over a certain region. We investigated the effect of this by measuring the detector efficiency with the point sources placed at various distances from the centre of exposure, in a plane perpendicular to the detector axis. The resulting curve, which was found to be energy independent, is shown in fig. 4 . This curve had to be convoluted with the lateral profile of the induced radioactivity in order to obtain the effective efficiency for the integral type of measurements. Since the lateral profile changes with the depth inside the stack, this effective efficiency will change slightly from plate to plate.

This brings us to a crucial point in the analysis. Accurate measurements of the lateral radioactivity profiles at various depths are not only needed to compute the effective detector efficiency, but also to be able to correct for particles that escaped detection. Obviously, this mainly concerns neutrons.

\subsection{Lateral profiles}

Lateral profiles were measured in various ways. First, we performed collimated measurements. An absorber with a hole in the middle was placed in between the detector and the plate to be measured. The size of the hole determined the granularity of the measurement. The plates were placed on an accurately movable support and the transverse profile of the radioactivity induced by the shower was recorded in a number of steps spanning a region that extends to a point where the measured signal is no longer significantly different from the background.

Many such measurements were done with a plastic scintillator counter sensitive to $\beta$ 's, the absorber being a $10 \mathrm{~mm}$ thick Al plate with a circular hole of $10 \mathrm{~mm}$ diameter in it. An example of the results of these measurements, performed at various depths, is shown in fig. 5. This technique is very useful to measure rapidly the global shower development in depth. In order to be able to determine the transverse profiles for radioactive products of different origin, e.g. uranium fission, spallation, or capture of slow neutrons, we also performed collimated $\gamma$-ray measurements with the 
Ge crystal in combination with a $5 \mathrm{~cm}$ thick lead absorber with a circular hole of $2 \mathrm{~cm}$ diameter in it. Figure 6a shows results of such measurements for $591 \mathrm{MeV}$ incident protons. Clearly, the transverse profiles are very different. Spallation products are strongly concentrated near the beam axis since they are predominantly produced by the primary beam particles. Fission products are also produced by these protons, but, moreover, by neutrons and, hence, the transverse profile of their concentration shows a tail that is not observed for the spallation products. The radioactive nuclei produced by slow neutron capture are, as expected, widely distributed over the stack.

Although $5 \mathrm{~cm}$ of lead is very effective in absorbing the $\gamma$-rays, it is not sufficient. This can be illustrated by a simple example. The region that we want to measure has a surface of $3 \mathrm{~cm}^{2}$. The whole plate has a surface of $2400 \mathrm{~cm}^{2}$. If the activity that we want to measure is homogeneously distributed over the plate, a transmission ('punch-through') of $10^{-3}$ would result in about equal numbers of $\gamma$ 's penetrating the lead shielding and those going through the hole. A similar situation occurs if one wants to measure at points in the tail of a strongly concentrated distribution. Of course, these effects are very strongly energy-dependent. The transmission of $\gamma$ 's through $5 \mathrm{~cm}$ of lead is $10^{-4}$ at $490 \mathrm{keV}, 10^{-3}$ at $600 \mathrm{keV}$, and $10^{-2}$ at $860 \mathrm{keV}$. For this reason, we also performed background measurements, in which the hole was closed. The plots of fig. $6 \mathrm{a}$ are corrected for this background.

Figure 6a might suggest that, given the size of the observed region $\left(20 \times 20 \mathrm{~cm}^{2}\right)$, the corrections for laterally escaping neutrons are negligibly small. This is, however, by no means the case. With this method, the lateral profiles of the concentration of radioactive products are obtained. In order to obtain absolute numbers of nuclei, these concentrations have in first order to be multiplied by the distance from the centre (the impact point of the beam). In fig. $6 \mathrm{~b}$ the same results are shown, translated into a number of nuclei as a function of this distance $r$. This leads to a completely different picture. Clearly, accurate measurements in the tail of the distributions are badly needed in order to perform reliable corrections. In the case of the uranium plates, the accuracy of the measurements was limited by the Compton background of high-energy $(\geq 1 \mathrm{MeV}) \gamma$-rays, amongst others from ${ }^{238} \mathrm{U}$ decay, scattering in the lead absorber. We found, nevertheless, a way to perform very accurate measurements of the radial radioactivity distribution. Fission products from uranium decay are produced with a considerable recoil energy $(\sim 100 \mathrm{MeV})$. If the fission occurs at the surface of a plate, the fission products may escape from the surface and penetrate up to several tens of microns into the next plate. If the next plate is made of non-radioactive material, measurements of fission products can be performed without any background from ${ }^{238} \mathrm{U}$ decay. Although only a small fraction of the fission products escape from the $3 \mathrm{~mm}$ uranium plates, we could measure very detailed spectra of fission products from the iron and scintillator plates inserted in some of the configurations that we irradiated. Because of the absence of background, many very weakly produced fission products, invisible in the uranium plates themselves, were found. For the purpose of transverse-distribution measurements we cut them into concentric circle arcs around the impact point, and measured these individually (fig. 7), a procedure that was not permitted for uranium plates for obvious safety reasons.

Figure 8 shows some results obtained from measurements on $500 \mu \mathrm{m}$ iron foils inserted in between the uranium plates. Fission product distributions are shown in fig. $8 \mathrm{~b}$, at various depths in the stack. Spallation and slow neutron capture in the iron itself also lead to easily observable radioactive products. Their distributions are given in figs. $8 \mathrm{a}$ and $8 \mathrm{c}$, for the same depths. All distributions agree with those obtained with the previously described method for the uranium plates, although their accuracy at large distances from the impact point is much better. The same was true for the results of the measurements on the scintillator plates. The measured lateral distributions were used to compute the effective detector efficiency for the integral type of measurements, and to correct the result for the fact that only a limited region of the plates was seen by the detector in these measurements. The procedures for this were as follows. The effective detector efficiency for a reaction product was obtained by convoluting its lateral distribution over the measured region $(r=0-11 \mathrm{~cm})$ with the curve of fig. 4 . This was done at various depths. The effective efficiencies for other depths were obtained by interpolation. Figure 9a shows the relation between the effective efficiency and the efficiency for a point source as a function of depth in the case of $591 \mathrm{MeV}$ protons on uranium.

The corrections for escaping neutrons were obtained with two methods. In the region where the measured radial distribution extends sufficiently far (covering $>95 \%$ of the total), the fraction of 
the activity produced in the $20 \times 20 \mathrm{~cm}^{2}$ region observed by the detector was computed numerically. At larger depth, and particularly beyond the last plate for which radial distributions were available, the corrections were obtained by fitting the measured lateral distributions to a theoretical formula (see next section for details), and computing from this fit the detected fraction of the activity. This was done at various depths, and the corrections to be applied for plates at other depths were obtained from interpolation. Figure $9 \mathrm{~b}$ shows the detected fraction of fission products and slow neutron captures as a function of depth for the $591 \mathrm{MeV} \mathrm{p-}{ }^{238} \mathrm{U}$ case. Especially deep inside the stack, the corrections for escaping neutrons are large. On the other hand, the contribution of plates at this depth to the total activity created in the shower development is small. It turns out that the detected fraction of fission products produced by the $591 \mathrm{MeV}$ protons (i.e. in the $20 \times 20 \mathrm{~cm}^{2}$ region observed by the detector), integrated over the full depth, amounts to about $70 \%$. For slow neutron capture by ${ }^{238} \mathrm{U}$ this fraction is lower and strongly dependent on the stack configuration. We found values between 40 and $65 \%$.

Although the corrections that had to be applied are substantial, the methods that we used to determine them are rather accurate and, therefore, the systematic uncertainties resulting from this are limited to only $10 \%$ of the overall correction that had to be applied. This estimate resulted from the comparison of the results from integral activity measurements for regions of various sizes and at various distances from the $\mathrm{Ge}$ detector.

\subsection{The number of ${ }^{238} U$ fissions}

In order to be able to convert the number of nuclei of a certain isotope, resulting from ${ }^{238} \mathrm{U}$ fission, to the number of ${ }^{238} \mathrm{U}$ fissions, one needs to know the branching ratio. It is known that the mass distribution of fission products depends, in principle, on the energy of the particle that induced the fission [17]. Slow neutrons, for example, tend to split the uranium nucleus into two asymmetric parts, because of nuclear-shell effects. If the fission is caused by fast particles (energy large compared to the nucleon binding energies) the resulting mass distribution is more symmetric. Therefore, in principle, one needs to know the energy spectrum of the particles that caused the fission to be able to make the conversion. The mentioned differences in mass yield are very important in the mass range $\mathrm{A}$ $\sim 110-130$. For nuclei around $A \sim 100$ and $A \sim 140$ the fission yield is much less sensitive to the production process (table 2 ). Therefore, only nuclides with masses around these values were used to compute the number of fissions. Table 2 also lists the fission products that were used to compute the number of fissions, together with the branching fractions that we adopted. The uncertainty in the energy spectrum of the particles that caused the fission leads to a systematic error in the number of fissions that we estimated to be $10 \%$. However, when comparing different stack configurations that were exposed to the same beam, this uncertainty largely disappears. It is a second-order effect, i.e. the difference between the energy spectra of the fission-inducing particles, which will lead to a systematic error in that case.

\section{EXPERIMENTAL RESULTS}

\subsection{Exposures with the $591 \mathrm{MeV}$ proton beam}

In the shower development of a multi-GeV hadron in a block of matter some fraction of the energy is used for $\pi^{0}$ production. These particles will develop electromagnetic showers. The mentioned fraction will fluctuate strongly from event to event. Its average rises with the energy of the incoming particle. The experimental information on this is, however, numerically, rather imprecise. Furthermore, as far as we know, no experimental data exist on the fission induced by $\pi^{0}$ showers in uranium. At some level photo-induced fission, e.g. via the giant nuclear resonance, should occur but since any nuclear process has to compete with the pair-production bremsstrahlung development of the electromagnetic shower (cross-section in the high-energy limit $\sim 65 \mathrm{~b}$ for uranium) the probability is small compared to fission induced by hadrons. For these reasons, the only way to measure the contribution of fission in the purely hadronic part of the shower is to use hadrons at an energy at which $\pi^{0}$ production does not play a role. This led to the choice of the CERN SC proton beam $(591 \mathrm{MeV})$ for our purpose. At this energy one cannot really speak of a hadron shower. A significant fraction of the protons $(26 \%)$ will lose their energy by ionization only and come to rest without any nuclear reaction. The other protons will interact with a uranium nucleus. In this process, a certain number of nucleons will be liberated, mainly neutrons because of the large neutron excess in 
the nucleus, and the Coulomb barrier for the protons. The nucleus may or may not fission in this process. The neutrons produced in this way (fission and/or spallation) may in turn create new fissions. This depends on their energy. The threshold for $\mathrm{n}$-induced ${ }^{238} \mathrm{U}$ fission is $\sim 1.5 \mathrm{MeV}$. A large fraction of the neutrons, especially the ones from spallation, will be produced at higher energy, but they may lose energy in a series of (in)elastic collisions, for which the cross-section is much larger than for fission. Moreover, there is a competition with other inelastic processes such as $(n, p),(n, \alpha)$, etc. Neutrons that do create fissions give rise to the production of new neutrons, which in turn may induce fission, etc. However, this sequence converges rapidly. The production of pions in the primary spallation reactions is insignificant at this energy. This can be concluded from the longitudinal profile of nuclei that can only be produced from spallation reactions. Such nuclei are abundantly found in the iron $\left({ }^{48} \mathrm{~V},{ }^{46 \mathrm{~m}} \mathrm{Sc},{ }^{51} \mathrm{Cr}\right)$ and scintillator $\left({ }^{7} \mathrm{Be}\right)$ plates from the calorimeter-type configurations. Their longitudinal distribution (very similar in all cases) is shown in fig. 10. The observed number of nuclei decreases gradually with increasing depth. After 1.5 nuclear interaction lengths, however, it suddenly drops by a factor of 7 over $1 \mathrm{~cm}$. This depth corresponds to the range of the protons. If pion production were considerable, this curve would look very different. The ionization loss by pions being much lower at this non-relativistic proton energy, their range is correspondingly larger and, therefore, the transition around $1.5 \lambda_{\mathrm{INT}}$ would be much less pronounced. Moreover, the curve from fig. 10 would tend to rise up to the point where interactions by pions produced earlier and new pions created balance. Pion production is more or less switched off.

For comparison, fig. 10 also shows the longitudinal distributions of ${ }^{103} \mathrm{Ru}$ nuclei, a representative ${ }^{238} \mathrm{U}$ fission product, and of ${ }^{239} \mathrm{~Np}$ nuclei, created via slow neutron capture, both measured in a massive uranium stack. The latter curve shows the features described before: a rise up to $\sim 0.3 \lambda_{\text {INT }}$ followed by a smooth decay and no transition near the proton range. This is not amazing: the nucleus concerned is only produced by neutrons. The curve for the fission products is a mixture of the two others. It rises initially, but still shows a discontinuity around the proton range, be it less pronounced than for spallation products. So the fission products are partially produced by proton-induced, and partially by neutron-induced reactions. Already from looking at this graph one gets quite a good impression of the answer to our question: how many fissions are caused by one particle? Each proton may at maximum cause one fission by itself, and a certain number of secondary (tertiary, etc.) fissions will come from neutrons produced in the nuclear reaction preceding this fission. The data from fig. 10 show that the proton-induced part is considerable. The neutron-induced part may be 5 or 10 times as large, but certainly not 50 .

The radial distributions of the three different classes of nuclei are also very different. They are shown in fig. 8. Spallation products are strongly concentrated near the beam axis; the nuclei from slow neutron capture have a broad distribution; relatively few are found near the beam axis, especially beyond $1 \lambda_{\mathrm{INT}}$. Fission products show a mixture of these two profiles. The proton-induced part is concentrated near the beam axis, the neutron-induced part has a much wider distribution. Beyond the proton range all distributions look similar. There are relatively few nuclei found near the beam axis, the maximum number of nuclei occurs at a distance from the axis that increases with depth, and this distance is larger for the nuclei created from thermal neutron capture than for the fission products. The reasons for this are clear. The radioactivity found beyond the proton range is predominantly produced by neutrons created near the beam axis from spallation reactions. Consider a point source that isotropically emits neutrons (fig. 11). The radial distribution of nuclei created by these neutrons measured in a flat plate at distance $d$ will then be:

$$
N(r)=r d /\left(r^{2}+d^{2}\right)^{3 / 2} \exp \left(-\sqrt{r^{2}+d^{2}} / \lambda_{n}\right),
$$

where $\lambda_{\mathrm{n}}$ is the average distance from the source at which the neutrons produced the nuclei concerned. This formula is only valid if there is no correlation between the neutron direction and $r$, which is true if the neutrons have elastically scattered before interacting. On an average this will be the case beyond the proton range (mean free path of neutrons in $U$ is only a few $\mathrm{cm}$ ), and it is practically always true for captured neutrons. If the neutrons have not scattered before interacting, which may happen close to the beam axis within the proton range, the formula becomes

$$
N(r)=r d /\left(r^{2}+d^{2}\right) \exp \left(-\sqrt{r^{2}+d^{2}} / \lambda_{n}\right) .
$$


If the neutrons are produced by a series of point sources, exponentially decreasing in intensity with depth up to the range, this model predicts for the radial distribution of nuclei at depth $\mathrm{d}$ :

$$
\mathrm{N}(\mathrm{r})=\int_{0}^{1.5 \lambda_{\mathrm{INT}}} \exp \left(-\mathrm{x} / \lambda_{\mathrm{INT}}\right) \mathrm{dx}\left[\mathrm{r}|\mathrm{d}-\mathrm{x}| /\left(\mathrm{r}^{2}+|\mathrm{d}-\mathrm{x}|^{2}\right)^{3 / 2}\right] \exp \left[-\sqrt{\mathrm{r}^{2}+(\mathrm{d}-\mathrm{x})^{2}} / \lambda_{\mathrm{n}}\right] .
$$

This formula, which contains only one parameter $\left(\lambda_{n}\right)$, gives a remarkably precise description of the distributions of nuclei produced by neutrons, as may be seen from the curves drawn in figs. 8 and 10.These curves were obtained from a simultaneous fit to all measured fission - or capture - data (longitudinal distribution + transverse distributions) beyond the proton range, using the same value of $\lambda_{\mathrm{n}}$. Best values for $\lambda_{\mathrm{n}}$ were found to be $14 \mathrm{~cm}$ for neutron-induced fission and $45 \mathrm{~cm}$ for neutron capture, in massive uranium. Especially, the distributions of nuclei in plates beyond the proton range, where only neutrons cause the nuclear reactions, are very well described. Therefore we used this model to compute the correction factors for escaping neutrons for the plates beyond the last one

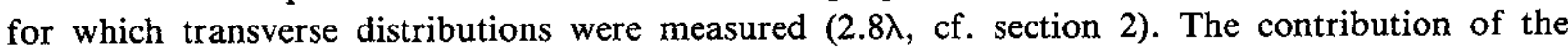
radioactivity in this region to the total is $<10 \%$ in all cases.

Within the proton range the agreement is less satisfactory. This is due to the fact that, close to the beam axis, there is some correlation between the neutron direction and the vector radius $\mathrm{r}$. Moreover, the fact that the beam itself has lateral dimensions plays a role.

\subsubsection{The number of fissions per particle}

Figure 12 shows the longitudinal distribution of ${ }^{140} \mathrm{Ba}$ nuclei , one of the fission products, for four different configurations:

a) massive ${ }^{238} U$

b) $3 \mathrm{~mm}{ }^{238} \mathrm{U}$, interleaved with $0.5 \mathrm{~mm} \mathrm{Fe}$

c) $3 \mathrm{~mm}{ }^{238} \mathrm{U}$, interleaved with $2.5 \mathrm{~mm}$ scintillator

d) $9 \mathrm{~mm}{ }^{238} \mathrm{U}$, interleaved with $2.5 \mathrm{~mm}$ scintillator.

The barium concentrations were measured in the uranium plates. The vertical scale gives the number of nuclei found per interaction length and per incoming proton (left-hand side). On the right-hand side, this scale is converted to the number of fissions per interaction length and per incoming proton, using the branching ratio from table 2 . The total number of fissions was found by integrating over the full depth. Similar plots were made for other fission products (table 2), of which the yield is relatively insensitive to the production mechanism (cf. section 2). The numbers of fissions found from the analysis of these different fission products were very consistent. The final values for the four different configurations are given in table 3. The errors quoted are almost entirely due to systematic uncertainties. The major sources of possible systematic effects are the ${ }^{238} \mathrm{U}$ fission branching ratios, the absolute number of protons, and escaping neutrons. Since these systematic effects largely cancel, ratios could be determined much more precisely than absolute numbers. In computing these ratios, we took into account that part of the energy is dissipated in the non $-{ }^{238} \mathrm{U}$ plates.

The results are very striking. The number of fissions per particle clearly depends on the stack configuration. No significant difference was observed between the massive ${ }^{238} \mathrm{U}$ and the $\mathrm{U} / \mathrm{Fe}$ configurations, but scintillator apparently reduces the number of fissions. This effect becomes stronger if more scintillator is added. There is also a clear difference between the slopes of the longitudinal profiles beyond the proton range (fig. 12). At large depth the fission product yield depends very strongly on the amount of scintillator present in the stack.

Part of the fissions are caused by protons. Both the longitudinal and radial distributions of fission products from this process are very different from those coming from neutron-induced fission. The distributions of proton-induced fission products are very similar to those observed for nuclei resulting from spallation reactions (cf. figs. $8 \mathrm{a}$ and 10). We made use of this to determine the fraction of proton-induced fission, in two mutually independent ways. With the first method the radial distribution of fission products in the first plate is analysed. Since the contribution of proton-induced fission is largest in this plate, the uncertainties concerning the shape of the neutron-induced distribution close to the beam axis, as discussed in section 2, are of minor importance. Once the number of proton-induced fissions in the first plate is known, it is known for all other plates, since the longitudinal distribution is the measured one for spallation products. The 
second method starts from the discontinuity observed in the longitudinal profile, near the proton range. This discontinuity tells us the concentration of proton-induced fission products near the range, and this allows us to compute the concentrations in the upstream plates in the same way as before. Figure 13 shows some results of this analysis. In fig. 13a, the measured longitudinal distribution of ${ }^{103} \mathrm{Ru}$ is shown, together with the proton-induced part. The curves from the two methods agree very well. In fig. 13b the radial distribution of these nuclei in iron and scintillator plates is compared. It is clear that the proton-induced fraction is relatively more important in the latter case. A numerical evaluation of these results, together with similar ones obtained for other fission products, leads to the conclusion that there is no significant difference between the number of proton-induced fissions per incoming particle in the various configurations. In fact this is a result that has to be expected, since the probability that the uranium nucleus fissions in the collision with a $591 \mathrm{MeV}$ proton is a constant of nature. We found it to be $48 \pm 6 \%$ (see table 3). The observed differences in the total number of fissions are entirely due to the neutron-induced part.

In section 2 we mentioned that the mass distribution of fission products depends on the energy of the particle that caused the uranium nucleus to fission. We saw a nice example of this in our data. We compared the yield of various fission products beyond the proton range and near the entrance of the stack. Figure 14 shows the ratio of both yields as a function of the atomic number A. If only slow neutrons were responsible for fission, all points would lie on a horizontal line. The huge dip around $A=120$ comes from fast protons, which tend to cause more symmetric fissions.

\subsubsection{The number of fissions per $\mathrm{GeV}$}

For calorimetric detection of high-energy hadron showers the relevant quantity is the number of fissions occurring per unit of energy $(\mathrm{GeV})$ for the purely hadronic part of the shower. In order to compute this quantity from the $591 \mathrm{MeV}$ proton data, one might take the results from table 3 and multiply by $1 / 0.591=1.7$. In doing so, however, one neglects the fact that these protons are non-relativistic and, hence, lose a considerable fraction of their energy by ionization. In fact $26 \%$ of the protons lose their energy by ionization only. From $\mathrm{dE} / \mathrm{dx}$ tables [18] we computed that the protons lose on an average $254 \mathrm{MeV}$, or $43 \%$ of their initial energy before interacting with a uranium nucleus. The energy transferred in nuclear reactions, i.e. available for liberating nuclear binding energy and giving kinetic energy to the evaporating nucleons and other nuclear fragments, amounts to $337 \mathrm{MeV}$, or $57 \%$ of the initial energy on an average. Multi-GeV hadrons lose a negligible fraction of their energy before the first nuclear interaction and hence it seems reasonable to convert the low-energy proton results into units per $\mathrm{GeV}$ using a multiplication factor $1 / 0.337=3.0$. In our opinion, this is not correct either, since, in later stages of the shower development of the high-energy hadron, important ionization losses will occur that are not accounted for in this way. The right value of the multiplication factor will be, therefore, somewhere in the interval 1.7-3.0. What one needs to know in order to determine a more precise number is the fraction of the purely hadronic energy (neglecting the $\pi^{0}$ component) that is not used for ionization, both at $591 \mathrm{MeV}$ and at high energy, $\mathrm{f}_{\mathrm{eff}}^{\mathrm{h}}(\mathrm{E})$. The multiplication factor to convert the $591 \mathrm{MeV}$ proton data into units per $\mathrm{GeV}$ will then to a good approximation be $(1 / 0.591) \mathrm{f}_{\text {eff }}^{\mathrm{h}}(\mathrm{E}$ high $) / \mathrm{f}_{\text {eff }}^{\mathrm{h}}(0.591)$.

Several published Monte Carlo calculations $[6,19]$ indicate that $\mathrm{f}_{\text {eff }}^{\text {h }} \sim 55-65 \%$ for high-energy hadron showers, which means that the calorimeter signal, due to the ionizing shower particles from the purely hadronic part (pions, protons) of the shower, amounts to $0.35-0.45$ of the minimum ionizing value, neglecting compensating effects.

For the $591 \mathrm{MeV}$ protons we cannot simply take $\mathrm{f}_{\mathrm{eff}}^{\mathrm{h}}(0.591)=0.57$, the value mentioned before. It is known that, quite often, such protons transfer only a small fraction of their energy in a peripheral interaction with the nucleus and then continue their way almost undisturbed. Moreover, in a central collision protons may be boiled off and lose their energy by ionization as well. Therefore, the total fraction of the energy going into ionization is definitely larger than the $43 \%$ calculated before. The first effect is clearly seen from the longitudinal distribution of spallation products (fig. 10). This distribution does not show a purely exponential behaviour with the interaction length as the slope. Compared to such an exponential less nuclei are found near the entrance of the beam, and somewhat more beyond $\sim 0.5 \lambda_{\mathrm{INT}}$. This experimental distribution of spallation products can be directly transferred into an energy distribution of the protons that caused the reaction. Moreover, the binding energy loss and the number of protons and neutrons created in this reaction are known, so 
the kinetic energy going into protons can be reasonably estimated. In doing so, we found that $\sim 55 \%$ of the initial proton energy goes into ionization, and $45 \%$ is used for liberating binding energy and giving kinetic energy to neutrons. So $\mathrm{f}_{\mathrm{eff}}^{\mathrm{h}}(0.591)=0.45 \pm 0.05$. The systematic error has to account for the fact that many different spallation reactions that are known to occur could not be investigated in this way.

Our estimate of the multiplication factor needed to convert the $591 \mathrm{MeV}$ proton results to units per $\mathrm{GeV}$ is, therefore

$$
(1 / 0.591) \times(60 \pm 5) /(45 \pm 5)=2.26 \pm 0.31
$$

The results are given in tables 3 and 4 .

\subsubsection{Slow neutron capture}

The differences between the distributions of nuclei created by slow neutron capture $\left({ }^{239} \mathrm{~Np}\right)$ in the various stack configurations studied are much more dramatic than those observed for fission products. Figure 15a shows the longitudinal distributions measured for the massive U, U/Fe and $3 \mathrm{~mm} \mathrm{U} /$ scintillator stacks. The profile observed in the stack containing scintillator is very different from the profiles observed in the other ones. The ${ }^{239} \mathrm{~Np}$ nuclei are on the average produced at much larger depth for the latter. The big impact of the scintillator on the neutrons that produce these nuclei is illustrated by fig. $15 \mathrm{~b}$. Here the longitudinal distribution of ${ }^{239} \mathrm{~Np}$ nuclei in the $9 \mathrm{~mm} \mathrm{U} / 2.5 \mathrm{~mm}$ scintillator stack is given. Three ${ }^{238} U$ plates were alternated with one scintillator plate. Huge plate-to-plate fluctuations in the ${ }^{239} \mathrm{~Np}$ concentration were observed. It turns out that the data fit to three smooth curves, for the first, second, and third plates following a scintillator plate.

Integration over the full longitudinal profile yielded the total number of ${ }^{239} \mathrm{~Np}$ nuclei, which corresponds in good approximation to the total number of (slow) neutrons per incoming proton. The results are given in table 4 . In spite of the fact that the distributions are very much different, the total number of neutrons measured is similar for the various configurations. The errors given are almost completely due to the uncertainties in the corrections for escaping neutrons which are large in this case (cf. section 2). Table 4 also gives the total number of slow neutrons per $\mathrm{GeV}$ of hadronic energy, computed in the same way as for the number of fissions. Concerning the errors given in table 4 , the same remarks that were made for table 3 apply. An additional source of errors, which also affects the ratios in this case, comes from the fact that the ${ }^{239} \mathrm{~Np}$ nuclei are apparently very inhomogeneously distributed in depth for the stacks that contain scintillator (cf. section 4.2).

\subsubsection{Comparison with lead}

We also exposed a block composed of lead bricks. Plates of depleted uranium $(3 \mathrm{~mm})$ were inserted after each layer of $\mathrm{Pb}(5 \mathrm{~cm})$. The uranium served to measure the neutron yield in lead via the creation of long-lived fission products and neutron capture. In fig. 16 the yields of fission products and ${ }^{239} \mathrm{~Np}$ are compared with those from massive uranium. Clearly, the neutron production per incoming proton is smaller in lead. The difference in the production of ${ }^{239} \mathrm{~Np}$ is larger than for the fission products. This is partly due to the fact that the protons themselves also create fission products in the uranium plates, at the same rate in both cases. After correcting for this effect, we found that the yield of neutrons that produce fission (i.e. the fast part of the neutron spectrum) is $53 \pm 5 \%$ of the massive uranium value. The production of ${ }^{239} \mathrm{~Np}$ (slow neutrons) is reduced to $33 \pm 3 \%$ (cf. tables 3 and 4 ). The errors are to a large extent due to the fact that only five uranium plates were inserted in the lead block.

From this, one may conclude not only that the total neutron yield in lead is considerably reduced with respect to uranium, but also that the neutron spectrum is harder in lead.

\subsection{Results from the $300 \mathrm{GeV}$ pion exposures}

Most of our efforts were concentrated on the analysis of fission products produced in a 7.3 nuclear interaction lengths thick massive uranium stack. Figure 17 shows the number of ${ }^{140} \mathrm{Ba}$ nuclei as a function of the depth in this stack. It is interesting to compare this distribution with its analogue at a 600 times lower energy (fig. 10). Although the way in which the incident particles lose their energy is very different, the curves clearly show some common features. They rise, reach a maximum, and then fall less steeply than they initially rose. There are also some clear differences. At 
$300 \mathrm{GeV}$, many (of the order of 100 ) pions will be produced in several generations of violent interactions. Their contribution to uranium fission will be relatively small near the entrance of the stack. Because of the large number of hadrons generated in the shower the contribution of fission induced by the incident particles themselves, which will be exponentially decreasing, cannot be more than $\sim 1 \%$. Therefore, the longitudinal profile of fig. 17 starts at a much lower value than that for the $591 \mathrm{MeV}$ protons. The discontinuity observed for these particles is absent in fig. 17 for obvious reasons, and the position of the maximum is, apart from the neutron interaction length, also determined by the shower development in this case and, therefore, occurs at a larger depth. From the $591 \mathrm{MeV}$ analysis we found that a considerable fraction of the fissions is proton-induced. Also at high energy fission directly induced by the interacting pions from the shower plays an important role. This can, for example, be seen from fig. 18a, which gives the production ratio between two fission products, ${ }^{140} \mathrm{Ba}$ and ${ }^{131} \mathrm{I}$, as a function of depth. The horizontal line corresponds to the literature value of this ratio, for fission induced by reactor neutrons. The experimental points are, except at the very beginning and deep inside the stack, situated well under this line, which means (cf., for example, fig. 14) that the fissions are on the average more symmetric than the ones induced by these neutrons and that high-energy particles therefore contribute significantly. Only deep inside the stack and near the front face (backward emitted) neutrons dominate. For comparison, fig. 18b shows the production ratio of two nuclides that differ by only one unit in mass. As expected, the effect is not measured in this case. The production ratio agrees well with the literature value, at each depth.

Integration over the longitudinal profile of fig. 16 yielded the total number of ${ }^{140} \mathrm{Ba}$ nuclei produced per incoming $300 \mathrm{GeV}$ pion. From this number the total number of fissions can be derived in the same way as described for the $591 \mathrm{MeV}$ protons. The average rate obtained from various fission products $\left({ }^{140} \mathrm{Ba},{ }^{103} \mathrm{Ru},{ }^{95} \mathrm{Zr}\right)$ was $1230 \pm 120$ fissions per particle, or $4.1 \pm 0.4$ fissions per $\mathrm{GeV}$. As before, the errors are almost completely due to systematic uncertainties (branching ratios of individual fission products, corrections for escaping neutrons). This number can be directly compared to the 9.8 fissions per $\mathrm{GeV}$ obtained for purely hadronic processes in massive uranium (table 4). We conclude that at $300 \mathrm{GeV} 58 \pm 7 \%$ of the energy is deposited in electromagnetic form ( $\pi^{0}$ showers). Strictly speaking, from this comparison we can only derive a lower limit, since photo-induced fission might also contribute to the overall 4.1 fissions per $\mathrm{GeV}$ observed. We tried to estimate this effect in the following way. In the energy range 6-20 MeV (the giant photo-nuclear resonance) photons from electromagnetic showers may cause photo-fissions. In an electromagnetic shower initiated by a GeV-type of photon many photons in this energy range will be generated. The cross-section for this process is considerable, at maximum $150 \mathrm{mb}$, but it has to compete with the $20 \mathrm{~b}$ cross-section for purely electromagnetic processes $\left(\mathrm{e}^{+} \mathrm{e}^{-}\right.$pair production, Compton scattering) in this energy region. Therefore, the $\gamma$ 's only traverse a small layer of matter before disappearing $(\sim 1 \mathrm{~cm})$.

We used the electromagnetic shower code EGS IV [20], in combination with the differential cross-sections from ref. [17] to compute the probability of photo-induced fission for a high-energy electromagnetic shower. The result was 0.09 fissions per $\mathrm{GeV}$. Therefore, of the 1230 fissions per particle that were experimentally observed, only $0.58 \times 300 \times 0.09 \sim 16$ fissions, or $\sim 1 \%$, are photo-induced. Other mechanisms, such as photo-fission via the neutron field in the pseudo-deuteron disintegration processes, or fission induced by neutrons from $(\gamma, n)$ reactions, will further increase this fraction, but it does not seem very likely that photons account for more than $10 \%$ of the total number of fissions observed.

It should be stressed that the fraction of the initial energy that goes into $\pi^{0}$ showers, as calculated in this way, directly relies upon the validity of the conversion of the low-energy data into units per GeV. The conversion factor (cf. section 3.1.2) partly relies upon Monte Carlo calculations, the validity of which we cannot judge. If we had not bothered about what happens beyond the first nuclear interaction, assuming that all processes scale with energy all the way from $0.591 \mathrm{GeV}$ to $300 \mathrm{GeV}$, and had just corrected for ionization losses before the first interaction, we would have found that $70 \%$ of the energy goes into $\pi^{0}$ showers at $300 \mathrm{GeV}$, instead of $58 \%$. Besides, all numbers per $\mathrm{GeV}$ given in tables 3 and 4 would go up by 30\%, yielding $\sim 13$ fissions per $\mathrm{GeV}$ and $\sim 60$ captured neutrons per $\mathrm{GeV}$.

In any case, a large fraction of the energy is deposited in the form of electromagnetic showers generated by $\pi^{0}$ decay. Our method, based on the occurrence of nuclear interactions, mainly probes 
the purely hadronic part of the shower. In order to be sensitive to this dominating component, we installed in one of our stacks an array of tiny radio-photoluminescence dosimeters around the beam axis in between two uranium plates at about the shower maximum (1.9 $\left.\lambda_{\text {INT }}\right)$. These dosimeters are, similarly to calorimeter readout systems, sensitive to all ionizing particles. Figure 19 shows, on a logarithmic scale, the total transverse shower profile (energy deposit per unit volume) and the transverse induced radioactivity profile. The tails of both distributions were fitted to each other. It is clear that close to the beam axis there is a huge hot spot of ionizing particles that do not cause nuclear reactions. The dosimeter that was sitting right on the beam axis recorded $700 \mathrm{krad}$ for $1.6 \times 10^{11}$ $300 \mathrm{GeV}$ pions. At $1.5 \mathrm{~cm}$ from the beam axis the dose had dropped by 2 orders of magnitude. In fact, the tiny yellow spot observed on our scintillators after a radiation resistance test triggered all the work described in this paper.

Although the contribution of photons from $\pi^{0}$ showers to the uranium fission is very limited, we did observe a nuclide which is, at least partly, produced by photonuclear reactions, namely ${ }^{237} \mathrm{U}$. It can be produced by photons in the same energy range as discussed before, via the reactions $(\gamma, n)$ and $(\gamma, p)$ on ${ }^{238} \mathrm{U}$. The EGS calculations combined with the $(\gamma, \mathrm{n})$ cross-section data from ref. [17] yield a probability of $0.23{ }^{237} \mathrm{U}$ nuclei produced via the giant resonance per $\mathrm{GeV}$ of electromagnetic energy, which would lead to a production of $0.58 \times 300 \times 0.23 \sim 40$ of such nuclei per $300 \mathrm{GeV}$ pion via this process alone. Production via pseudo-deuteron disintegration [16] and $(\gamma, p)$ reactions will further increase this yield to a value which anyway is larger than for a typical fission product (30-40 per incoming pion). Purely hadronic ways to produce this uranium isotope will be dominated by the $(\mathrm{n}, 2 \mathrm{n})$ reaction (cross-section $\sim 1 \mathrm{~b}$, threshold $6 \mathrm{MeV})$. Other reactions, such as $(\mathrm{p}, \mathrm{d}),(\pi, \mathrm{n})$ have cross-sections that are 2 orders of magnitude lower. Experimentally, we observed $\sim 150$ of these nuclei per incoming pion. Both from their transverse distribution (fig. 20) and their longitudinal distribution (fig. 21), we conclude that photoproduction plays a significant role. The transverse distribution is clearly much more concentrated near the beam axis than for fission products (fig. 20). The longitudinal distribution shows a kink that occurs after about $3-4 \mathrm{~cm}$ in the uranium (see insert of fig. 21a). Before the kink the curve rises much more steeply than the equivalent one for fission products (fig. 21b). No hadronic process can cause such a kink, the typical distance for interactions being $\sim 10 \mathrm{~cm}$, so that a smooth behaviour has to be expected. If the beam, while entering the stack, has a high-energy $\gamma$ component, this will develop an electromagnetic shower that reaches a maximum after about 10 radiation lengths (i.e. the position of the kink) and then decreases. Pions that undergo charge exchange in the low-Z material immediately upstream of the target $\left(\sim 0.1 \lambda_{\text {INT }}\right)$ might cause these phenomena, which would then account for $\sim 3 \%$ of the total ${ }^{237} \mathrm{U}$ production (unfolding the left part of the curve). Beyond $5 \lambda_{\text {INT }}$, where the production of new $\pi^{0}$ 's is very small, the ${ }^{237} U$ yield drops much faster than the one for fission products, and reaches at large depth the value that can be expected from $(\mathrm{n}, 2 \mathrm{n})$ reactions caused by fission neutrons (fig. $21 \mathrm{~b}$ ).

As in the case of the $591 \mathrm{MeV}$ protons, inserting scintillator plates had dramatic effects on the results. Figure 22 shows some of these effects. The measurements were done with the $\beta$-counter set-up, and therefore do not allow one to distinguish between the various kinds of activities. Figure $22 \mathrm{c}$ shows the transverse radioactivity concentration profile measured at the same depth $\left(0.7 \lambda_{\mathrm{INT}}\right)$ for massive ${ }^{238} \mathrm{U}$ and for a stack that had one scintillator plate inserted after each tenth uranium plate. The latter profile is significantly broader. Figure $22 \mathrm{~b}$ shows how the transverse radioactivity profile develops when the shower goes through only two scintillator plates inserted at the shower maximum $\left(1.9 \lambda_{\mathrm{INT}}\right)$ in a massive ${ }^{238} \mathrm{U}$ stack. These results are consistent with the picture that emerged from the more detailed $591 \mathrm{MeV}$ measurements. Scintillator plates will increase the ratio of ${ }^{239} \mathrm{~Np}$ to fission production, and since ${ }^{239} \mathrm{~Np}$ has a broader transverse profile, the lateral distribution of the total induced radioactivity broadens. Figure 22a shows the tails of the longitudinal profiles for massive $\mathrm{U}$ and a $\mathrm{U} / \mathrm{Cu} /$ scintillator stack. The activity in the latter decreases much faster in the region (3-6) $\lambda_{\mathrm{INT}}$ (cf. also figs. 12 and 15a).

\section{DISCUSSION}

We first summarize the results of our analysis.

i) The number of fissions created by the purely hadronic part of a high-energy hadronic shower amounts to about 10 per $\mathrm{GeV}$ in massive uranium.

ii) About $10 \%$ of these fissions are induced by charged particles, the rest by neutrons. 
iii) The neutron-induced part decreases considerably $(\sim 20 \%)$ if scintillator plates are introduced in between the uranium plates. Introduction of iron (to simulate liquid-argon readout) does not produce such an effect.

iv) Only a small fraction of the neutrons created in the shower cause uranium fission, at maximum $20 \%$ (massive uranium).

v) The total number of neutrons is not very sensitive to the calorimeter composition. It amounts to $\sim 45$ per $\mathrm{GeV}$ of hadronic energy.

vi) In stacks that contain plastic scintillator plates the neutrons are captured much closer to the point where they are produced than in massive uranium and uranium/iron stacks.

vii) The total neutron yield in lead is about $60 \%$ lower than in ${ }^{238} \mathrm{U}$. These neutrons are, however, on an average more energetic.

viii) Pions at $300 \mathrm{GeV}$ that enter a block of uranium lose $\sim 60 \%$ of their energy in electromagnetic form, and create $\sim 4$ fissions per $\mathrm{GeV}$.

\subsection{A simple model}

In order to induce ${ }^{238} \mathrm{U}$ fission, a neutron should have sufficient energy. Figure 23a shows the fission cross-section as a function of energy [17]. This cross-section decreases very steeply for neutron energies below $1.5 \mathrm{MeV}$. In the same graph the total cross-section is shown. It turns out that in the energy range 1-10 MeV other reactions, mainly elastic and inelastic scattering, are at least ten times more likely than fission. Figure $23 \mathrm{~b}$ shows the Maxwellian energy spectrum of neutrons resulting from ${ }^{238} \mathrm{U}$ fission. About 2.5-4 neutrons are produced per fission, depending on the energy of the incident particle. Fission induced by neutrons that are themselves created from ${ }^{238} \mathrm{U}$ fission yields on an average 2.8 new neutrons. A considerable fraction of the neutrons is, however, produced at energies below the fission threshold. The others are very likely to lose energy in another way before creating fission (fig. 23a) and, in doing so, drop below this threshold as well. Neutrons from spallation reactions are also predominantly resulting from nuclear evaporation [16]. Their energy spectra are, in general, a bit harder than those from fig. 22b, but also here a large fraction of the neutrons will lose energy by (in)elastic scattering processes and become incapable of causing fission. It is, therefore, not amazing that the number of neutrons that we measured is much larger than the number of fissions.

We used the Rudstam formula $[16,21]$ to compute the cross-sections for the different spallation reactions that may occur if $591 \mathrm{MeV}$ protons hit ${ }^{238} \mathrm{U}$ nuclei. The distribution of the number of spallation neutrons produced in these processes is given in fig. 24. The average number is 10 . Suppose a fraction $f$ of these induce ${ }^{238} \mathrm{U}$ fission, each of which yields 2.8 new neutrons, of which a fraction $\mathrm{f}^{\prime}$ create new fissions, etc. The total number of neutron-induced fissions per incoming proton is then $10 f\left[1+2.8 f^{\prime}\left\{1+2.8 f^{\prime}+\left(2.8 f^{\prime}\right)^{2} \ldots\right\}\right]$, the number of neutrons that do not induce fission and are finally captured will be $10(1-f)+10 f \times 2.8\left(1-f^{\prime}\right)\left[1+2.8 f^{\prime}+\left(2.8 f^{\prime}\right)^{2}+\ldots\right]+0.5 \times 4(1-f)$, with $f>f^{\prime}$. The last term is due to the p-induced fission that eventually followed the primary interaction. Figure 25 shows the relation between $\mathrm{f}, \mathrm{f}^{\prime}$, and the number of neutrons that induce fission or are captured. The experimental values obtained for massive uranium give a point in this graph that fits very well in the region predicted by this simple calculation. Apparently $f$ is of the order of 0.2 and $\mathrm{f}^{\prime} \sim 0.1$, which means that $\sim 50 \%$ of the $\mathrm{n}$-induced fissions come from spaliation neutrons, the rest from higher-order processes. For lead, no neutron multiplication through fission occurs. Therefore, the number of neutron-induced fissions in the uranium plates inserted in the lead block will simply be $10 \mathrm{f}$, and the number of neutrons being captured $10(1-\mathrm{f})$. The results are given by the dashed curve in fig. 25 . The fact that our experimental point for lead is located slightly to the left-hand side of this curve might indicate that, in the spallation reactions on lead, on an average less neutrons are produced than the 10 computed for uranium. If we had taken 8 we would have found a curve for lead which runs almost parallel to the dashed one displayed in fig. 25 and passes nicely through our experimental point. The $f$ value agrees with the one found for uranium, too. Such a reduction in the number of spallation neutrons is plausible because of the smaller $n / p$ ratio in the nucleus, the larger binding energy per nucleon, and the reduced Coulomb barrier for evaporating protons.

This very simple description explains quantitatively why the ratio captures/fissions observed in the uranium plates inserted in the lead block is so much lower than in the massive uranium case. The 
difference comes from the soft fission neutrons. The total number of neutrons produced in lead is about a factor of 3 smaller than in uranium, but their total energy is only $\sim 50 \%$ lower.

\subsection{Effects of the scintillator}

We now switch to the scintillator. Figure 23 shows that, in the energy region where the neutrons are produced, the probability of (in)elastic scattering is an order of magnitude larger than fission. Therefore, the neutrons that eventually induce fission, have on an average been scattered many times before doing so. In the previous section we showed that the fission yield in massive ${ }^{238} \mathrm{U}$ is considerably influenced by this effect, since only $\sim 20 \%$ of the spallation neutrons cause fission, while a much larger fraction is produced above the fission threshold. Elastic scattering is the most likely process. However, in uranium, it takes many such processes to bring the energy down since, on an average, the neutron loses only 1/239 part of its energy per collision. Inelastic scattering is, therefore, in massive ${ }^{238} \mathrm{U}$ probably the main source of neutron energy loss (see fig. 23a). This situation changes drastically if plastic scintillator is inserted in the stack. Elastic collisions with the protons in the scintillator (chemical composition $\mathrm{C}_{5} \mathrm{H}_{8} \mathrm{O}_{2}$ ) take away, on an average, half of the neutron energy per collision. Since the average energy loss per collision increases, more neutrons will drop below the fission threshold by these collisions and less fissions will occur. This is what we observed. Assuming that the energy loss from elastic collisions is proportional to $\mathrm{N} \cdot \sigma_{\mathrm{el}} \cdot 1 /(\mathrm{A}+1)$, where $\mathrm{N}$ is the number of target nuclei per unit surface with atomic number $\mathrm{A}$ and cross-section for elastic scatterring $\sigma_{\mathrm{el}}$, we computed that it is increased by a factor $\sim 100$ if $2.5 \mathrm{~mm}$ scintillator plates are inserted in between the $3 \mathrm{~mm}$ uranium plates. The $0.5 \mathrm{~mm}$ Fe plates increase the energy loss due to elastic collisions only by $30 \%$.

Figure 26 shows the cross-section for neutron capture. This cross-section sharply rises for very low neutron energy, the $(\mathrm{n}, \gamma)$ resonance region. Before being captured, neutrons have on an average lost almost all of their energy. If we now look at fig. $15 \mathrm{a}$, we see that the neutrons are captured much closer to the region where they are actually produced, i.e. the first $10 \mathrm{~cm}$, if the stack contains scintillator. The effect of iron is small. The scintillator is clearly very effective in slowing them down to an energy at which they can easily be captured. The phenomena observed in fig. $15 \mathrm{~b}$ indicate that many neutrons are slowed down into the resonant absorption region by the scintillator. Because of the isotropic neutron direction, one would to first order expect the results for two uranium plates facing the same scintillator plate to be the same. Differences up to $15 \%$ were experimentally observed. This can only be explained if the distribution of ${ }^{239} \mathrm{~Np}$ in one plate is very inhomogeneous. The $\gamma$ 's from ${ }^{239} \mathrm{~Np}$ are low in energy $(278 \mathrm{keV}$ ) and hence suffer from self-absorption in the $3 \mathrm{~mm}$ uranium plate (see fig. 3). All plates were measured with their downstream face towards the detector. The largest ${ }^{239} \mathrm{~Np}$ concentrations were systematically measured in the plates for which this side neighboured a scintillator plate. Inhomogeneities at this length scale ( $\mathrm{mm}$ ) can only be caused by resonant absorption $\left(10^{2}-10^{3} \mathrm{~b}\right)$.

Figure 15a also shows that the moderation by the scintillator has the effect that less neutrons escape from the stack geometry. This was in fact already observed during the exposure to the beam. The neutron monitors in the beam area indicated only half the value when the ${ }^{238} \mathrm{U}$ target was replaced by the fine-sampling uranium/scintillator stack.

Another consequence of the neutron moderation by the scintillator can be seen from fig. 12. In the region beyond the proton range, fission is mainly caused by neutrons created upstream. Whether or not these neutrons have sufficient energy to cause fission deep inside the stack will strongly depend on the amount of scintillator that they have to traverse. Therefore, the longitudinal distribution of fission products in this region decreases much faster in the uranium scintillator stack than in the massive uranium one.

All these effects should, in principle, also occur if iron instead of scintillator is added to the uranium. However, since the increase in the energy loss from elastic collisions is 300 times smaller than for scintillator, the differences from massive uranium are marginal.

\subsection{Consequences for calorimetry}

In a sampling calorimeter the hadron energy is measured from the ionization of the active medium, either in a direct (e.g. liquid argon) or indirect (scintillation light) way. Contrarily to electromagnetic showers a large fraction, typically one half, of the energy is not produced in the form 
of ionizing particles in a purely hadronic shower. In order to make the calorimeter response to a realistic high-energy hadron shower independent of the energy fraction taken by $\pi^{0}$ 's, the response to the hadronic component needs to be increased with respect to what can be expected from the ionizing part of the shower particles. Neutrons offer this possibility. They may be inelastically scattered, captured, or cause fission. In all cases $\gamma$-rays are produced which may contribute to the measured signal. In another paper [10] we will show, however, that a calorimeter with high-Z absorber material is very inefficient in detecting $\gamma$ 's in the energy range around $1 \mathrm{MeV}$. The signal is reduced by a factor $\sim 2.5$ with respect to minimum ionizing particles at the same energy. Much more interesting is the direct contribution of neutrons to the detected signal. If the active medium contains free protons, these will recoil in elastic neutron collisions and, hence, ionize the medium. This is an effect that will occur for scintillator or warm liquid (TMP) readout, but not for liquid argon.

The present paper shows how important scintillator is for this purpose. It turns out that if scintillator is added to uranium it largely determines the energy loss mechanism of neutrons. In other words, a large fraction of the neutron energy is directly transferred to the protons in the scintillator and, therefore, has to increase the calorimeter signal. Let us make an estimate of this effect, for the $3 \mathrm{~mm} \mathrm{U} / 2.5 \mathrm{~mm}$ scintillator considered here. We measured a neutron production of $\sim 45$ per $\mathrm{GeV}$, say with an average energy of $5 \mathrm{MeV}$. So, $\sim 25 \%$ of the total hadronic energy is going into neutrons. Let us assume that half of this energy is lost in elastic collisions, probably a realistic estimate considering the huge effect of scintillator on the total energy loss pattern. Then, $125 \mathrm{MeV} / \mathrm{GeV}$ is going into elastic neutron collisions. The calculations mentioned in the previous section show that $90 \%$ of this energy, or $110 \mathrm{MeV} / \mathrm{GeV}$, is transferred to free protons in the scintillator. The range of these recoil protons is very small compared to the thickness of the scintillator plates and, therefore, almost all of this energy is directly transferred into a measurable signal.

These $110 \mathrm{MeV} / \mathrm{GeV}$ have to be compared with the energy deposit in the scintillator by the ionizing particies. The sampling fraction of this calorimeter being $8.5 \%$ for minimum ionizing particles we find $\sim 40 \mathrm{MeV} / \mathrm{GeV}$ for this. The contribution of the neutrons, in terms of energy deposit in the active medium, is huge! In this example, it is much larger than the energy deposited by the charged shower particles. Since the recoil protons are very densely ionizing particles, saturation effects in the scintillator will occur. These effects, determined by the kB factor [22], will determine how much the calorimeter signal (the amount of light produced) will increase because of the neutrons. Experimental $\mathrm{kB}$ values [23] indicate that an increase of the order of $50 \%$ is feasible.

A very interesting aspect is that the relative contribution of neutrons to the signal of uranium hadron calorimeters sensitively depends on the sampling width. This can easily be seen as follows. Suppose we increase the uranium plate thickness by a factor of 2; the energy deposited by charged particles from the shower development in the scintillator will then be half of its previous value. The neutrons, however, still transfer most of their energy lost in elastic collisions to the protons in the scintillator, and therefore their relative contribution to the calorimeter signal is larger than before. Calorimeters with a small sampling fraction may, therefore, show overcompensation, which was in fact experimentally observed [24]. The e/ $\pi$ ratio may be tuned to the optimal value, using the sampling fraction as a parameter. In ref. [10] we work this out quantitatively. We also show that it should, in principle, be possible to build a lead/scintillator calorimeter with equal response to electromagnetic and hadronic showers. Using the experimental data on neutron production from the present study we find that such a calorimeter should have a lower sampling fraction than current devices.

Liquid-argon calorimeters do not benefit from neutrons, since elastic scattering does not yield an observable signal. On the other hand, our results show that the number of fissions is about $20 \%$ larger than in a uranium/scintillator calorimeter. Since each fission yields on an average $7.4 \mathrm{MeV}$ in the form of prompt $\gamma$-rays, the total energy of these fission $\gamma$-rays amounts to $75 \mathrm{MeV} / \mathrm{GeV}$ for uranium/liquid argon and $60 \mathrm{MeV} / \mathrm{GeV}$ for uranium/scintillator.

Another important source of $\gamma$-rays is $\left(n, n^{\prime} \gamma\right)$ reactions. In liquid-argon calorimeters the neutrons that do not cause uranium fission will lose practically all their kinetic energy via this process (cross-section $\sim 2.5 \mathrm{~b}$ in the few $\mathrm{MeV}$ region), since the energy loss per elastic collision $(\sigma \sim 5 \mathrm{~b})$ is negligibly small. Taking again on average $5 \mathrm{MeV}$ per neutron we find $\sim 230 \mathrm{MeV} / \mathrm{GeV}$ in the form of soft $\gamma$ 's from this source. With scintillator readout this will be much less, since here energy losses via elastic collisions dominate, as we saw before. 
The major source of $\gamma$-rays, however, are $(\mathrm{n}, \gamma)$ reactions. Each captured neutron liberates the binding energy of $\sim 6 \mathrm{MeV}$ in the form of $\gamma$-rays, which yields the impressive total of $\sim 270 \mathrm{MeV} / \mathrm{GeV}$, independent of the type of readout chosen. However, since the neutrons, on an average, have to be slowed down to energies in the (k)eV range before being captured (fig. 26), this is a rather slow process. Brueckmann [25] has calculated that it may take up to $1 \mu$ s to be sufficiently efficient to $\gamma$ 's from this source. After $100 \mathrm{~ns}$, which is a typical signal integration time in calorimeters, $\sim 25 \%$ of the neutrons would be captured, according to his calculations. If these calculations are correct, the signal integration time might be used to tune the response of the calorimeter to these $\gamma$ 's. So in total, in uranium/liquid-argon calorimeters $370-570 \mathrm{MeV} / \mathrm{GeV}$ is available in the form of soft $\gamma$-rays; for scintillator readout it is $\sim 130 \mathrm{MeV}$ less. Ionizing particles from the hadron shower deposit $\sim 400 \mathrm{MeV} / \mathrm{GeV}$. For liquid-argon calorimeters, therefore, an increase of the hadronic signal due to $\gamma$-rays of $25-45 \%$ can be expected, depending on the fraction of the captured neutrons that contribute to the signal, and in spite of the calorimeter inefficiency for detecting these $\gamma$ 's.

Unlike for neutrons, the contribution of $\gamma$ 's to the hadronic signal does not vary, to first order, with the sampling fraction. Unless one adds hydrogen containing admixtures to the argon, the only way to tune the response is via the signal integration time. It remains questionable whether the (small?) signal range available in this way is sufficient to reach the signal equalization regime for practical devices.

\section{SUMMARY AND CONCLUSIONS}

We have experimentally determined how many fissions are produced when a high-energy hadron penetrates a block of matter containing ${ }^{238} \mathrm{U}$ that is sufficiently large to stop all the strongly interacting particles created in this process, including the neutrons. We were able to distinguish between neutron-induced fission and fission induced by the charged particles from the shower development, the latter being responsible for $\sim 10-15 \%$ of the fissions, depending on the stack configuration. In addition, we measured the total neutron yield in the shower development, which turned out to be 4-7 times larger than the number of fissions, also depending on the composition of the calorimeter block. Plastic scintillator turned out to have an important moderating effect on the neutrons and, therefore, the results obtained for uranium/scintillator configurations were very different from the ones obtained for $\mathrm{U} /$ iron ( $\simeq$ liquid argon) and massive uranium. Less fissions are produced and more neutrons are detected in a given volume in the scintillator case. We also measured neutron production in a massive lead block. It is about $60 \%$ lower than in uranium, but the neutrons are on an average faster. This is due to the absence of fission neutrons in lead. The fission yield in high-energy pion showers was measured to be $4.1 \pm 0.4$ fissions per GeV. Combining the results of the measurements at low and high energy, we could extract the average fraction of the energy spent on $\pi^{0}$ production in the latter case. These measurements also give a good impression of the calorimeter depth needed to detect such high-energy particles with a good energy resolution (7-8 nuclear interaction lengths). The results from these measurements are of crucial importance for understanding uranium hadron calorimetry. Calorimeters employing scintillator readout clearly offer the possibility of equalizing the response to electromagnetic and hadronic showers and hence achieve the best possible energy resolution, by choosing the appropriate sampling fraction for the type of scintillator used. The same is true for calorimeters that use other active media, provided that these contain a significant fraction of free protons. The possibilities of uranium/liquid-argon calorimeters seem to be less promising in this respect.

\section{Acknowledgements}

We are very much indebted to the CERN radiation protection group, who generously placed their facilities at our disposal, and in particular to G. Roubaud, who performed many measurements for us. Without the continuous support and encouragement of C. Fabjan and W. Willis, these experiments would not have been possible. We thank $\mathrm{H}$. Specht for many interesting discussions. 


\section{REFERENCES}

[1] C.W. Fabjan et al., Nucl. Instrum. Methods 141 (1977) 61;

T. Akesson et al., Nucl. Instrum. Methods A241 (1985) 17.

[2] F. Corriveau, CERN HELIOS Int. Note 145 (1986), unpublished.

[3] B. Cox, Uranium-liquid argon calorimetry; preliminary results from the DO tests, presented at the DPF Meeting of the American Physical Society, University of Oregon, Eugene, Oreg., 1985, FERMILAB-Conference 86/14-E.

D. Hitlin, SLD liquid argon prototype tests, in Proc. Workshop on Compensated Calorimetry, Pasadena, 1985, CALT-68-1305.

[4] C.W. Fabjan and W.J. Willis, in Proc. Calorimeter Workshop, Batavia, 1975 (ed. M. Atac) (FNAL, Batavia, Ill., 1975).

[5] S. Iwata, Nagoya University Report, DPNU-3-79 (1979).

[6] U. Amaldi, Phys. Scripta 23 (1981) 409.

[7] C.W. Fabjan and T. Ludlam, Annu. Rev. Nucl. Part. Sci. 32 (1982) 335.

[8] C.W. Fabjan, Lectures given at the NATO Advanced Studies Institute on Techniques and Concepts in High Energy Physics (II), St. Croix, Virgin Islands, 1984.

[9] W.J. Willis, Proc. Discussion Meeting on HERA Experiments, Geneva, 1984, DESY-HERA 85/01, p. 499.

[10] Y. Sirois and R. Wigmans, On the energy resolution of uranium and other hadron calorimeters, to be published.

[11] T.W. Armstrong et al., An investigation of fission models for high energy radiation transport calculations, Kernforschungsanlage Jülich GmbH, Jül-1859, 1983, and references therein.

[12] W.A. Gibson et al., in Electronuclear Division Annual Progress Report for Period ending 31 December 1965 (ORNL-3940) (Oak Ridge National Laboratory, Tenn., 1966), p. 110.

[13] L.R. Veeser et al., Nucl. Instrum. Methods 117 (1974) 509.

[14] T.A. Gabriel, Nucl. Instrum. Methods 150 (1978) 145.

[15] E. Storm and H.J. Israel, Nuclear Data Tables 7 (1970) 565.

[16] M. Barbier, Induced radioactivity (North Holland, Amsterdam, 1969).

[17] V.M. Gorbachev, Y.S. Zamyathin and A.A. Lbov, Nuclear reactions in heavy elements, a data handbook (Pergamon Press, London, 1980), and references therein.

[18] J.F. Janni, At. Data Nucl. Data Tables 27 (1982) 341.

[19] A. Baroncelli, Nucl. Instrum. Methods 118 (1974) 445.

[20] W.R. Nelson, H. Hirayama and D.W.O. Royers, The EGS4 Code System, SLAC-Report-165 (1985).

[21] G. Rudstam, Philos. Mag. 46 (1955) 344;

G. Rudstam, Z. Naturforsch. 21a (1966) 7.

[22] J.B. Birks, Scintillation counters (McGraw Hill, New York, 1953).

[23] R.L. Craun and D.L. Smith, Nucl. Instrum. Methods 80 (1970) 239 and references therein.

[24] M. De Vincenzi et al., Nucl. Instrum. Methods A243 (1986) 348.

[25] H. Brueckmann, Hadron calorimetry - a puzzle of physics, in Proc. Workshop on Compensated Calorimetry, Pasadena, 1985, CALT-68-1305. 
Table 1

Properties of nuclides produced in the shower development of high-energy hadrons in ${ }^{238} \mathrm{U}$, used for this analysis

\begin{tabular}{|c|c|c|c|}
\hline Nuclide & $\begin{array}{c}\text { Decay } \\
\text { constant } \lambda \\
\left(\mathrm{d}^{-1}\right)\end{array}$ & $\begin{array}{c}\gamma \text { energy } \\
(\mathrm{keV})\end{array}$ & $\begin{array}{c}\gamma \text { branching } \\
\text { ratio } \\
(\%)\end{array}$ \\
\hline${ }^{95} \mathrm{Zr}$ & 0.0108 & $\begin{array}{l}724.2 \\
756.7\end{array}$ & $\begin{array}{l}43.7 \\
55.4\end{array}$ \\
\hline${ }^{99} \mathrm{Mo}$ & 0.252 & 140.5 & 90.7 \\
\hline${ }^{103} \mathrm{Ru}$ & 0.0177 & 497.1 & 89.5 \\
\hline${ }^{115} \mathrm{Cd}$ & 0.311 & 336.2 & 49.7 \\
\hline${ }^{120} \mathrm{Sb}$ & 0.120 & $\begin{array}{l}1023.3 \\
1171.7\end{array}$ & $\begin{array}{r}99.0 \\
100.0\end{array}$ \\
\hline${ }^{122} \mathrm{Sb}$ & 0.257 & 564.0 & 70.8 \\
\hline${ }^{124} \mathrm{Sb}$ & 0.0115 & $\begin{array}{r}602.7 \\
1691.0\end{array}$ & $\begin{array}{l}98.4 \\
49.0\end{array}$ \\
\hline${ }^{126} \mathrm{Sb}$ & 0.0559 & $\begin{array}{l}414.8 \\
695.0\end{array}$ & $\begin{array}{r}98.0 \\
100.0\end{array}$ \\
\hline${ }^{127} \mathrm{Sb}$ & 0.180 & $\begin{array}{l}473.0 \\
685.7\end{array}$ & $\begin{array}{l}24.7 \\
35.3\end{array}$ \\
\hline${ }^{131} \mathrm{I}$ & 0.0864 & 364.5 & 81.2 \\
\hline${ }^{132} \mathrm{Te} / \mathrm{I}$ & 0.213 & $\begin{array}{r}228.2 \\
522.6 \\
667.7 \\
772.6 \\
954.6 \\
1398.6\end{array}$ & $\begin{array}{r}88.1 \\
16.1 \\
99.0 \\
76.4 \\
18.1 \\
7.1\end{array}$ \\
\hline${ }^{140} \mathrm{Ba} / \mathrm{La}$ & 0.0544 & $\begin{array}{r}328.8 \\
487.0 \\
537.3 \\
815.8 \\
1596.5\end{array}$ & $\begin{array}{l}20.7 \\
45.9 \\
24.4 \\
23.6 \\
95.4\end{array}$ \\
\hline${ }^{141} \mathrm{Ce}$ & 0.0213 & 145.4 & 48.4 \\
\hline${ }^{143} \mathrm{Ce}$ & 0.504 & 293.3 & 42.8 \\
\hline${ }^{237} U$ & 0.103 & 208.0 & 21.7 \\
\hline${ }^{239} \mathrm{~Np}$ & 0.294 & 277.6 & 14.5 \\
\hline
\end{tabular}


Table 2

Yield of some individual ${ }^{238} \mathrm{U}$ fission products,

produced by particles of different energy (data taken from ref. [17]).

\begin{tabular}{|c|c|c|c|c|}
\hline Nuclide & $\begin{array}{l}\text { Yield from slow } \\
\text { neutron-induced } \\
\text { fission } \\
(\%)\end{array}$ & $\begin{array}{l}\text { Yield from fast } \\
\text { particle-induced } \\
\text { fission } \\
(\%)\end{array}$ & $\begin{array}{l}\text { Used in } \\
\text { analysis }\end{array}$ & $\begin{array}{c}\text { Adopted } \\
\text { yield }\end{array}$ \\
\hline${ }^{89} \mathrm{Sr}$ & $4.4 \pm 0.4$ & $3.0 \pm 0.1$ & & \\
\hline${ }^{99} \mathrm{Mo}$ & $7.0 \pm 0.7$ & $6.6 \pm 0.5$ & $\mathrm{X}$ & $6.8 \pm 0.7$ \\
\hline${ }^{103} \mathrm{Ru}$ & $3.9 \pm 0.5$ & $3.4 \pm 0.3$ & $\mathrm{X}$ & $3.7 \pm 0.4$ \\
\hline${ }^{106} \mathrm{Ru}$ & $2.85 \pm 0.30$ & $3.0 \pm 0.2$ & & \\
\hline${ }^{111} \mathrm{Ag}$ & $0.094 \pm 0.012$ & $1.88 \pm 0.66$ & & \\
\hline${ }^{112} \mathrm{Pd}$ & 0.07 & $1.14 \pm 0.08$ & & \\
\hline${ }^{113} \mathrm{Ag}$ & 0.04 & $1.21 \pm 0.06$ & & \\
\hline${ }^{115} \mathrm{Cd}$ & $0.046 \pm 0.007$ & $1.15 \pm 0.05$ & & \\
\hline${ }^{117} \mathrm{Cd}$ & 0.031 & $1.04 \pm 0.04$ & & \\
\hline${ }^{127} \mathrm{Sb}$ & $0.17 \pm 0.02$ & $1.49 \pm 0.09$ & & \\
\hline${ }^{131} I$ & $3.5 \pm 0.3$ & $4.6 \pm 0.2$ & $\mathrm{X}$ & $4.0 \pm 0.4$ \\
\hline${ }^{132} \mathrm{Te}$ & \pm 0.4 & $4.3 \pm 0.1$ & $\mathrm{x}$ & $4.2 \pm 0.4$ \\
\hline${ }^{140} \mathrm{Ba}$ & \pm 0.5 & $4.8 \pm 0.2$ & $\mathrm{x}$ & $5.0 \pm 0.5$ \\
\hline${ }^{143} \mathrm{Ce}$ & 4.89 & $3.6 \pm 0.1$ & & \\
\hline
\end{tabular}


Table 3

Number of fissions generated per $591 \mathrm{MeV}$ proton and per $\mathrm{GeV}$ hadronic energy deposit, for various calorimeter configurations.

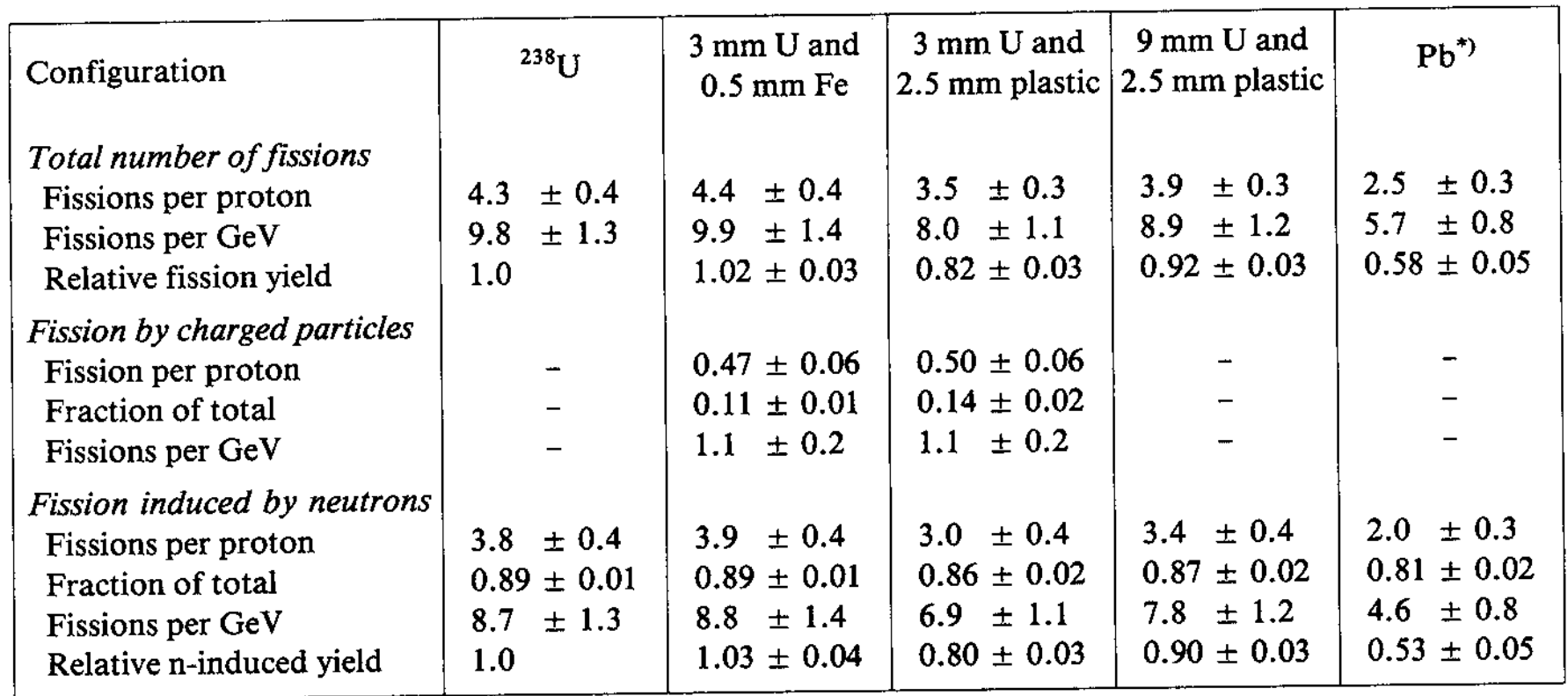

*) This column represents the number of fissions observed in thin uranium plates inserted in a massive lead block (see text).

Table 4

Number of neutrons captured per $591 \mathrm{MeV}$ proton and per $\mathrm{GeV}$ hadronic energy deposit, for various calorimeter configurations.

\begin{tabular}{|c|c|c|c|c|c|}
\hline Configuration & ${ }^{238} \mathrm{U}$ & $\begin{array}{c}3 \mathrm{~mm} \mathrm{U} \text { and } \\
0.5 \mathrm{~mm} \mathrm{Fe}\end{array}$ & $\begin{array}{c}3 \mathrm{~mm} \mathrm{U} \text { and } \\
2.5 \mathrm{~mm} \text { plastic }\end{array}$ & $\begin{array}{c}9 \mathrm{~mm} \mathrm{U} \text { and } \\
2.5 \mathrm{~mm} \text { plastic }\end{array}$ & $\left.\mathrm{Pb}^{*}\right)$ \\
\hline Neutrons captured per proton & $19.2 \pm 2$ & $18.7 \pm 2$ & $21 \pm 3$ & $23 \pm 5$ & $6.1 \pm 1.0$ \\
\hline Neutrons captured per $\mathrm{GeV}$ & $43 \pm 7$ & \pm 7 & $47 \quad \pm 8$ & $51 \pm 13$ & \pm 3 \\
\hline Relative capture yield & 1.0 & $0.97 \pm 0.05$ & $1.09 \pm 0.10$ & $1.18 \pm 0.26$ & $0.32 \pm 0.05$ \\
\hline $\begin{array}{l}\text { Ratio of neutron-induced } \\
\text { fissions to neutron captures }\end{array}$ & $0.20 \pm 0.01$ & $0.21 \pm 0.01$ & $0.14 \pm 0.01$ & $0.15 \pm 0.02$ & $0.33 \pm 0.03$ \\
\hline
\end{tabular}

*) This column represents the number of fissions observed in thin uranium plates inserted in a massive lead block (see text). 
Fig. 1 Experimental set-up for the $\gamma$-ray measurements of the radioactivity induced by the shower in one of the plates from a (uranium) stack.

Fig. 2 The distribution of the fission product ${ }^{99} \mathrm{Mo}$ created in a $300 \mathrm{GeV} \pi^{-}$shower, as a function of depth in the stack.

Fig. 3 Absorption of $\gamma$-rays on their way to the detector. The transmission out of the $3 \mathrm{~mm}$ uranium plate and through the $2 \mathrm{~cm}$ wooden support is plotted as a function of energy.

Fig. 4 Geometrical effects on the detection efficiency of $\gamma$-rays (see text).

Fig. 5 Transverse profile of radioactivity induced by $300 \mathrm{GeV} \pi^{-}$showers in a massive ${ }^{238} \mathrm{U}$ stack measured at various depths with a $\beta$-counter.

Fig. 6 a) Transverse profiles of the distributions of nuclei created in uranium by spallation, fission, or neutron capture at $0.1 \lambda_{\text {INT }}$ depth, from $591 \mathrm{MeV}$ protons; b) the same data converted into numbers of nuclei as a function of the distance to the impact point (radial distributions). The curves are drawn to guide the eye.

Fig. 7 A direct measurement of the radial distributions of nuclei for the same plate as fig. 6 .

Fig. 8 Distributions of the number of nuclei, as a function of the distance to the impact point of a $591 \mathrm{MeV}$ proton beam, measured in the $3 \mathrm{~mm}{ }^{238} \mathrm{U} / 0.5 \mathrm{~mm}$ Fe stack, at various depths; a) for spallation products; b) for fission products; c) for products from neutron capture.

Fig. 9 The effective efficiency for detecting various products created by $591 \mathrm{MeV}$ protons in ${ }^{238} \mathrm{U}$, as a function of depth: a) geometrical effects, due to the non-pointlike distribution; b) the detected fraction of nuclides that are produced by neutrons that might escape.

Fig. 10 Distribution of spallation, fission, and capture products, created by $591 \mathrm{MeV}$ protons, as a function of depth, in massive ${ }^{238} \mathrm{U}$.

Fig. 11 A simple model to compute the radial distribution of radioactive nuclei.

Fig. 12 Distribution of ${ }^{140} \mathrm{Ba}$ nuclei created by $591 \mathrm{MeV}$ protons, as a function of depth in various stack configurations.

Fig. 13 a) Decomposition of the measured longitudinal ${ }^{103} \mathrm{Ru}$ distribution into a $591 \mathrm{MeV}$ proton-induced part and a neutron-induced part. The decomposition was made using the discontinuity near the proton range (curve 1) or the radial distribution in the first plate (curve 2). b) The radial distributions of ${ }^{103} \mathrm{Ru}$ nuclei measured in the first plates of uranium/scintillator and uranium/iron stacks exposed to $591 \mathrm{MeV}$ protons.

Fig. 14 The ratio of the fission product yields measured beyond the range of the $591 \mathrm{MeV}$ protons, and in the first few uranium plates, as a function of the atomic mass, normalized to ${ }^{140} \mathrm{Ba}$.

Fig. 15 a) The longitudinal distributions of ${ }^{239} \mathrm{~Np}$ nuclei (from neutron capture) produced by $591 \mathrm{MeV}$ protons in various stack configurations; b) same as (a), for a $9 \mathrm{~mm}{ }^{238} \mathrm{U} / 2.5 \mathrm{~mm}$ scintillator configuration. The three curves are drawn through points that correspond to measurements of the first, second, and third $3 \mathrm{~mm}$ uranium plates downstream of a scintillator plate. The arrows indicate the position of scintillator plates.

Fig. 16 Comparison of longitudinal distributions of nuclei produced by $591 \mathrm{MeV}$ protons in lead and uranium (absolute scale): a) nuclei from neutron capture; b) fission products.

Fig. 17 Distribution of ${ }^{140} \mathrm{Ba}$ nuclei produced by $300 \mathrm{GeV} \pi^{-}$showers in a massive ${ }^{238} \mathrm{U}$ stack, as a function of depth.

Fig. 18 a) The ratio of the yields of two fission products, ${ }^{140} \mathrm{Ba}$ and ${ }^{131} \mathrm{I}$, produced by $300 \mathrm{GeV} \pi^{-}$ showers in massive ${ }^{138} \mathrm{U}$, as a function of depth; b) the same for ${ }^{131} \mathrm{I}$ and ${ }^{132} \mathrm{Te}$.

Fig. 19 The transverse profile of the total energy deposit by a $300 \mathrm{GeV} \pi^{-}$shower in massive ${ }^{238} \mathrm{U}$, measured with a dosimeter array, and the induced $\beta$-radioactivity profile, measured at the same depth (1.9 $\lambda$, near the shower maximum). The tails of both distributions are fitted to each other (logarithmic scale).

Fig. 20 A comparison of the transverse profiles of ${ }^{237} \mathrm{U},{ }^{239} \mathrm{~Np}$, and fission products produced by $300 \mathrm{GeV} \pi^{-}$showers in massive ${ }^{238} \mathrm{U}$, at $1.0 \lambda$.

Fig. 21 a) The longitudinal distribution of ${ }^{237} \mathrm{U}$ nuclei from the $300 \mathrm{GeV} \pi^{-}$exposure; b) the ratio of ${ }^{237} \mathrm{U}$ to ${ }^{140} \mathrm{Ba}$ as a function of depth. 
Fig. 22 Some effects of scintillator plates on the $\beta$-radioactivity created by $300 \mathrm{GeV} \pi^{-}$showers in ${ }^{238} \mathrm{U}$ stacks. a) Comparison of the transverse profiles for massive uranium and $30 \mathrm{~mm}$ uranium $/ 2.5 \mathrm{~mm}$ scintillator, at $0.7 \lambda_{\mathrm{INT}}$. b) The effect of one scintillator plate in a massive uranium stack on the radioactivity profiles. c) Comparison of the longitudinal distributions in massive uranium and a stack consisting of uranium up to $3.5 \lambda_{\mathrm{INT}}$, and a mixture of uranium, scintillator, and copper plates beyond this.

Fig. 23 a) The cross-section for neutron-induced ${ }^{238} \mathrm{U}$ fission as a function of energy. b) Neutron energy spectra from ${ }^{238} \mathrm{U}$ fission.

Fig. 24 The number of neutrons from ${ }^{238} \mathrm{U}$ spallation reactions induced by $591 \mathrm{MeV}$ protons.

Fig. 25 The relation between the number of neutrons that produce fission and the number of neutrons being captured, together with model predictions (see text).

Fig. 26 The cross-section for neutron capture in ${ }^{238} \mathrm{U}$. 


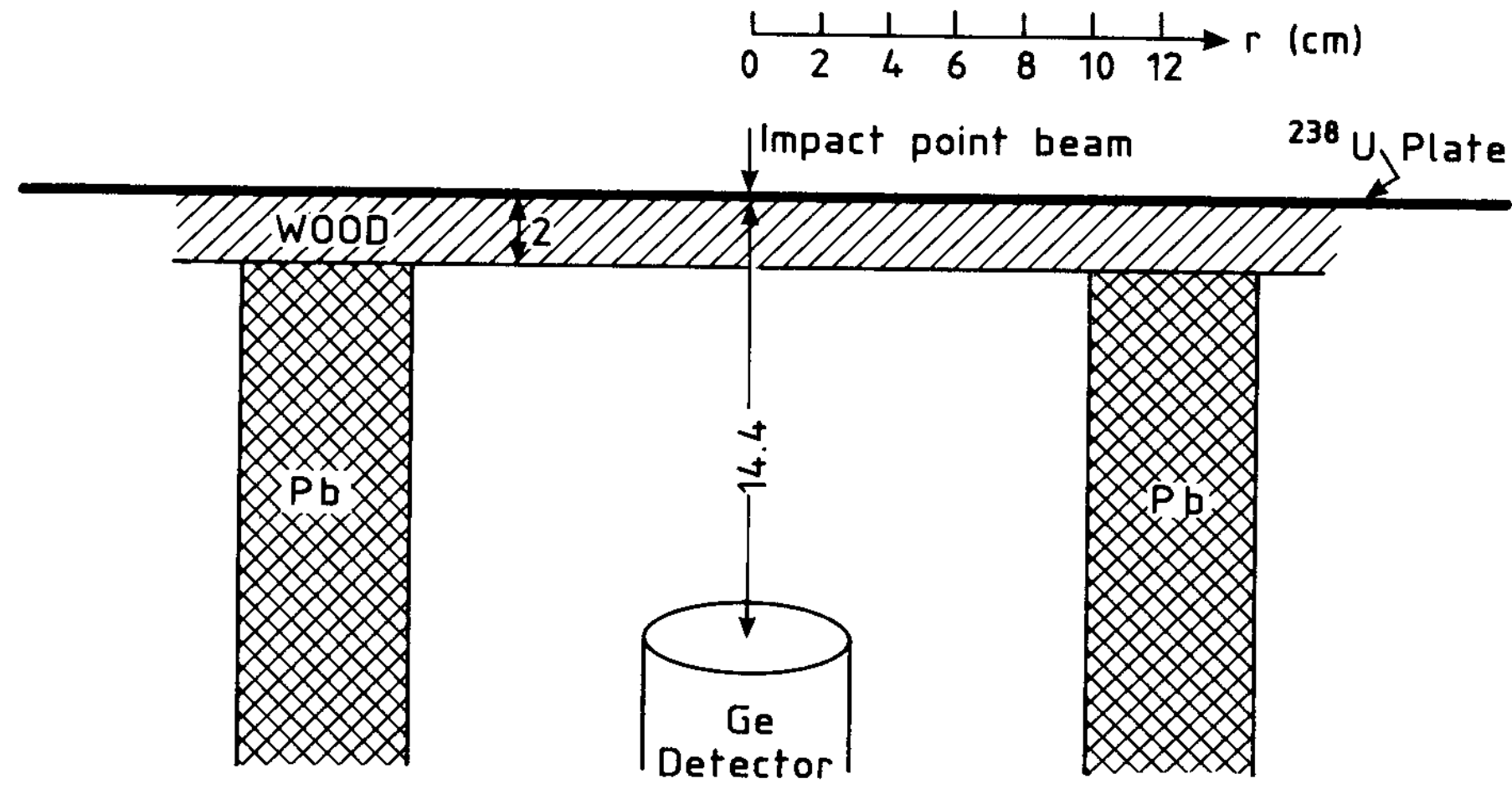

Fig. 1 


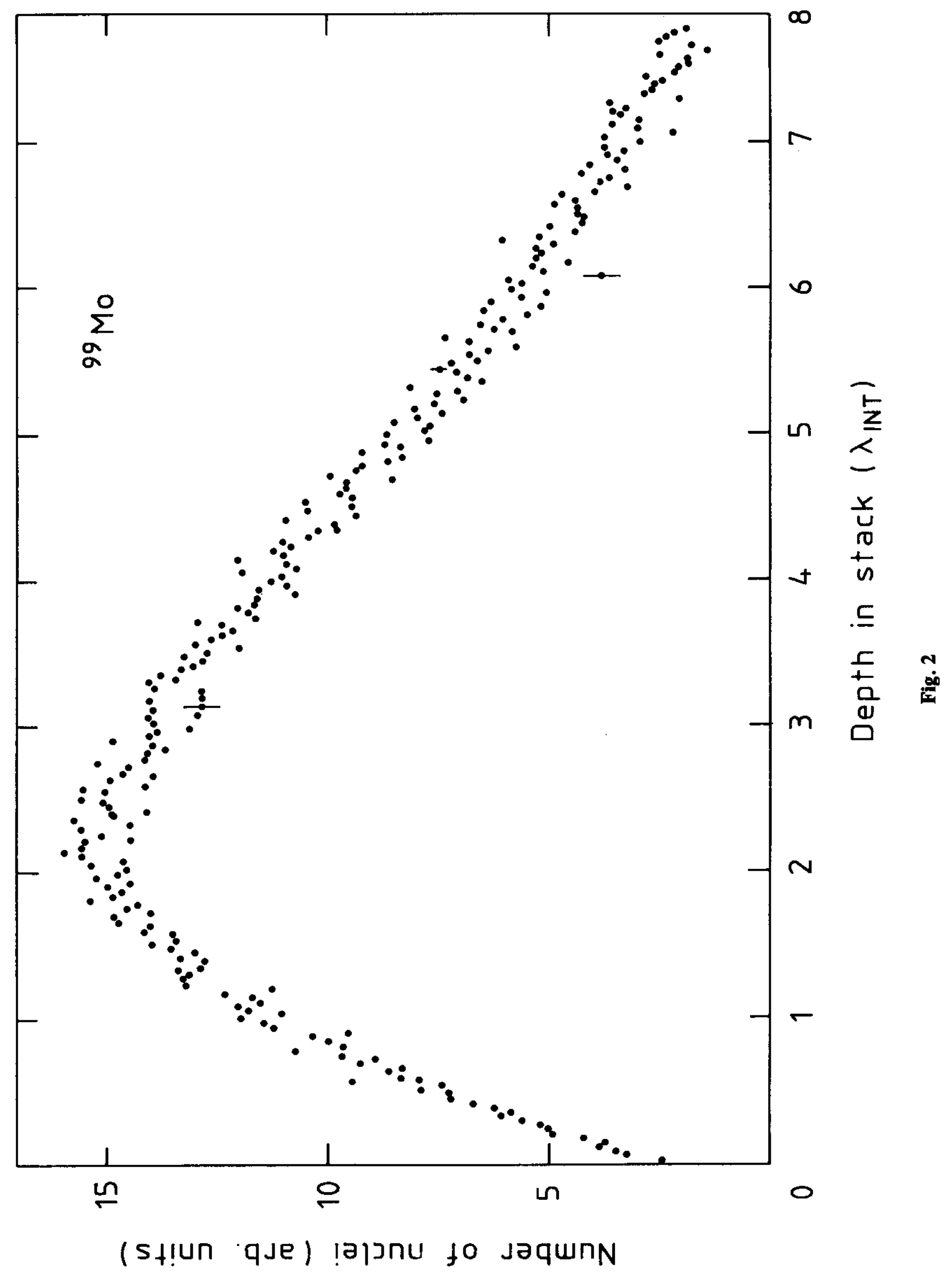




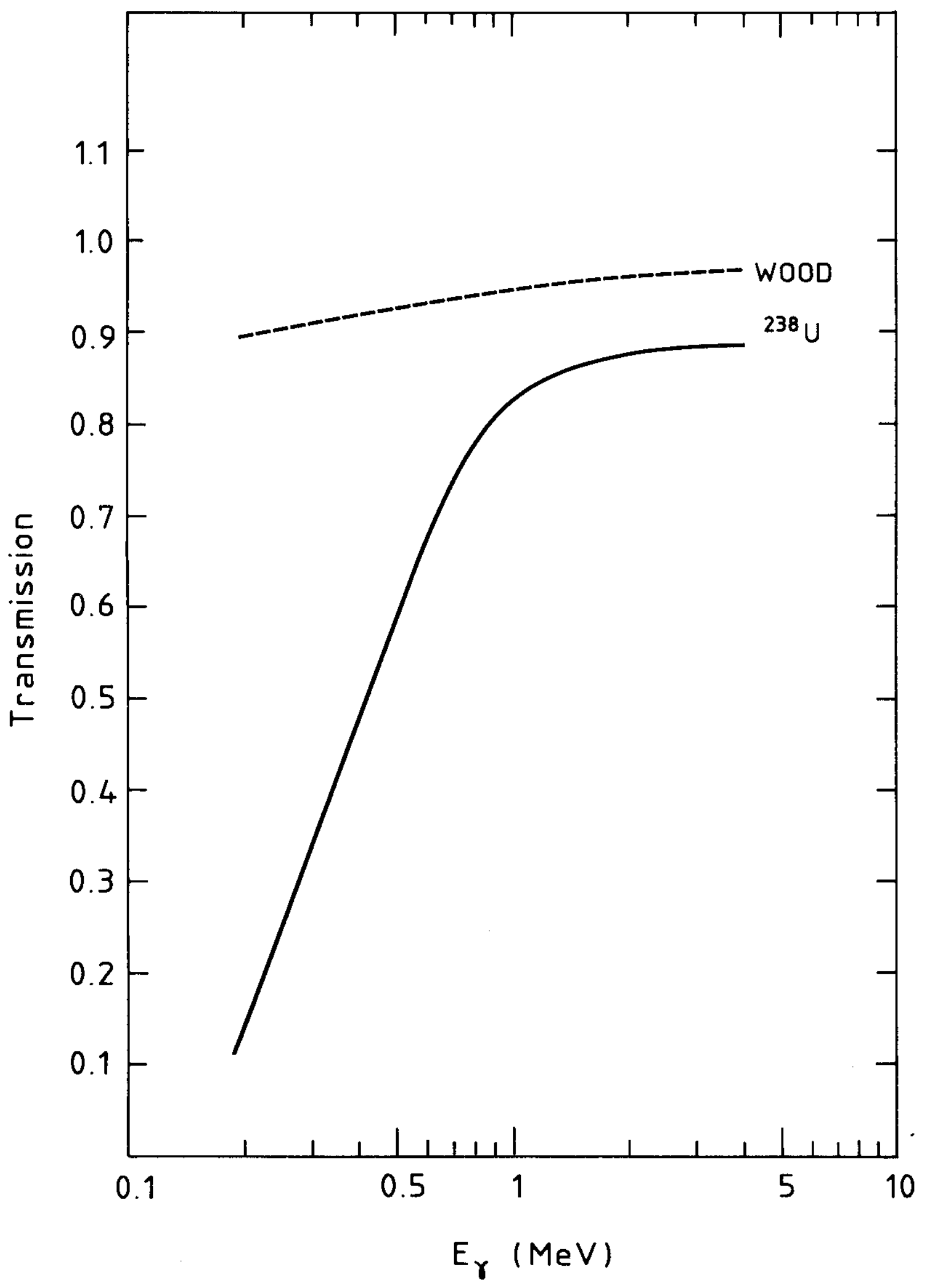

Fig. 3 


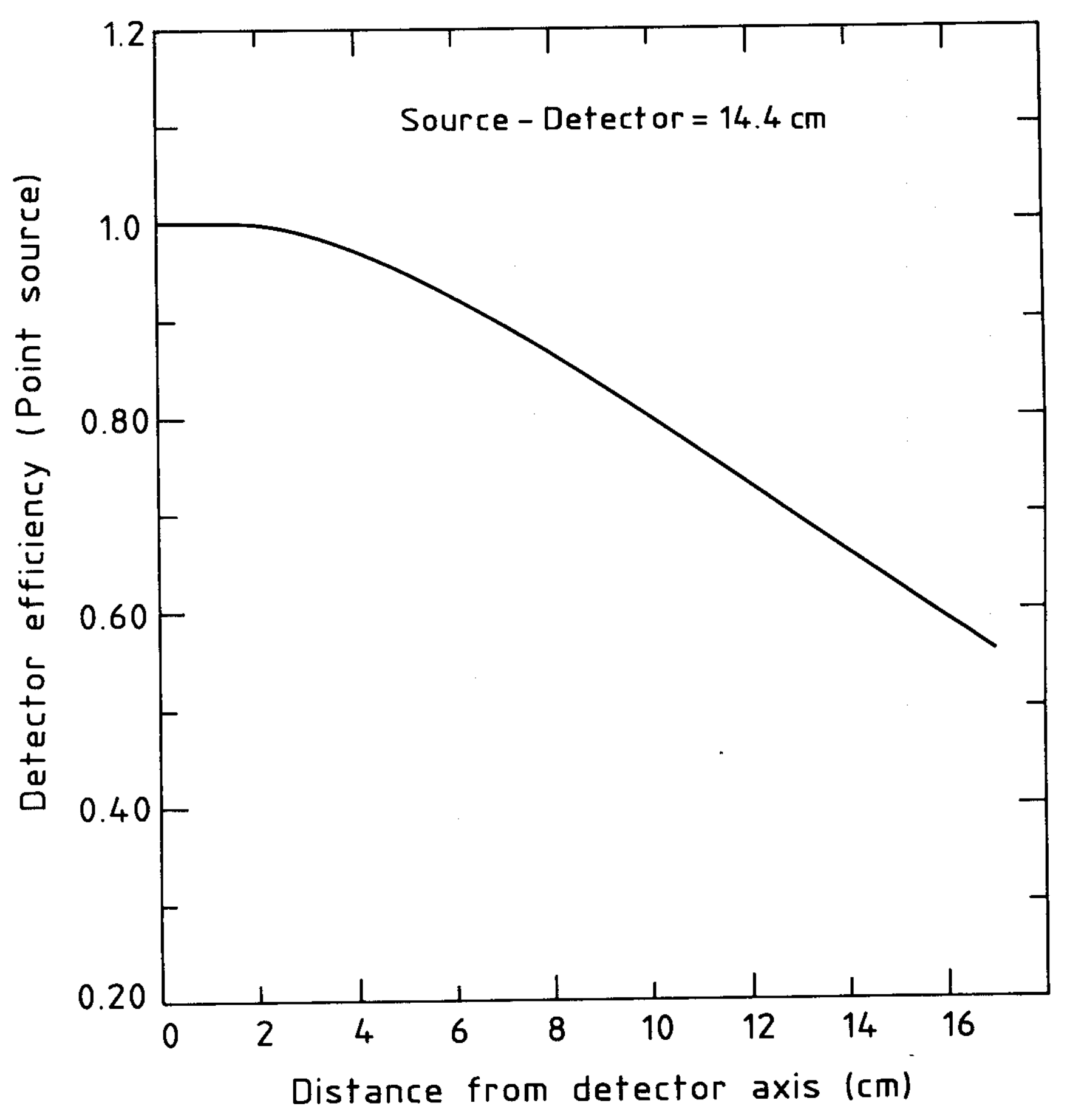

Fig. 4 


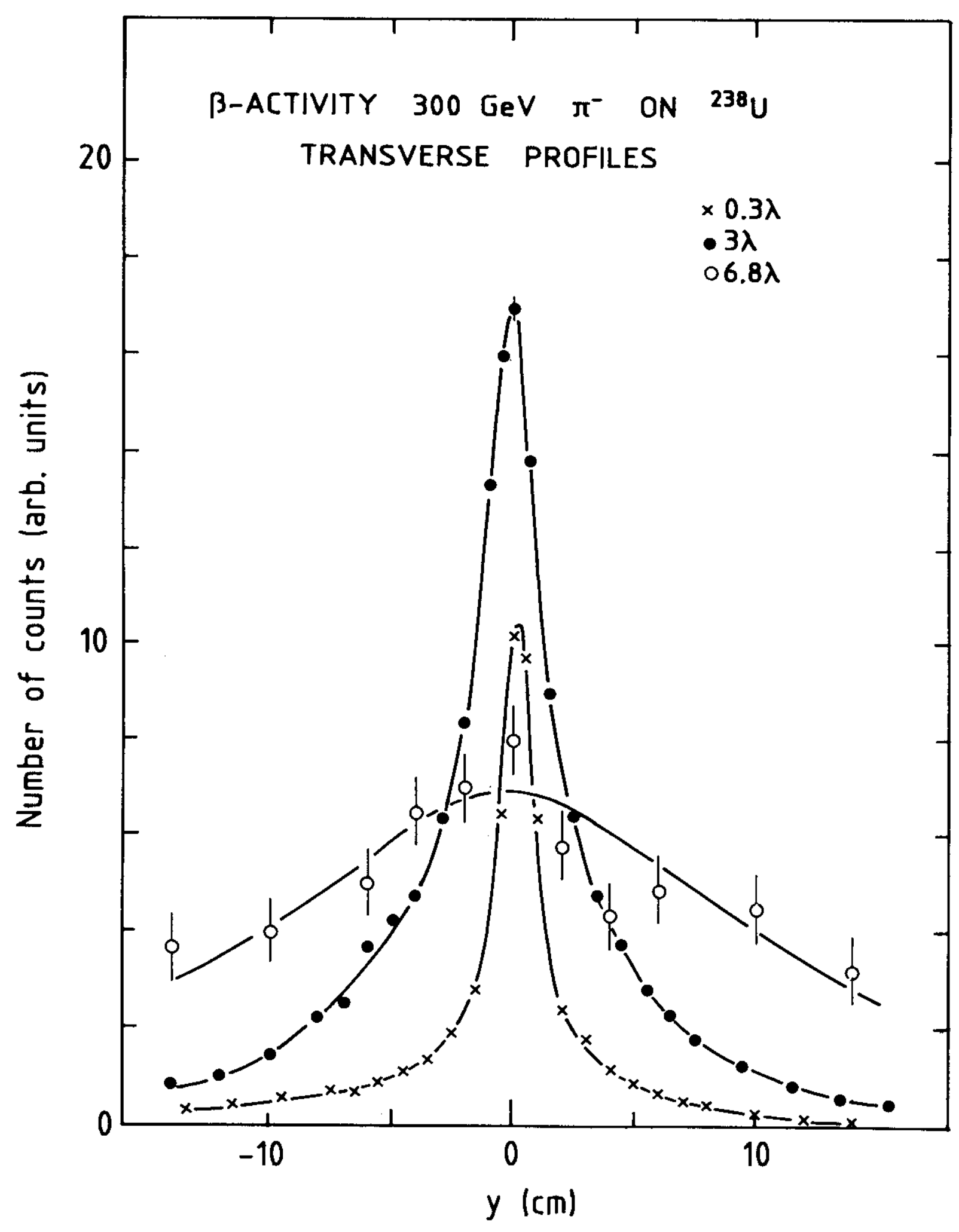

Fig. 5 
Number of nuclei/cm (arb. units)
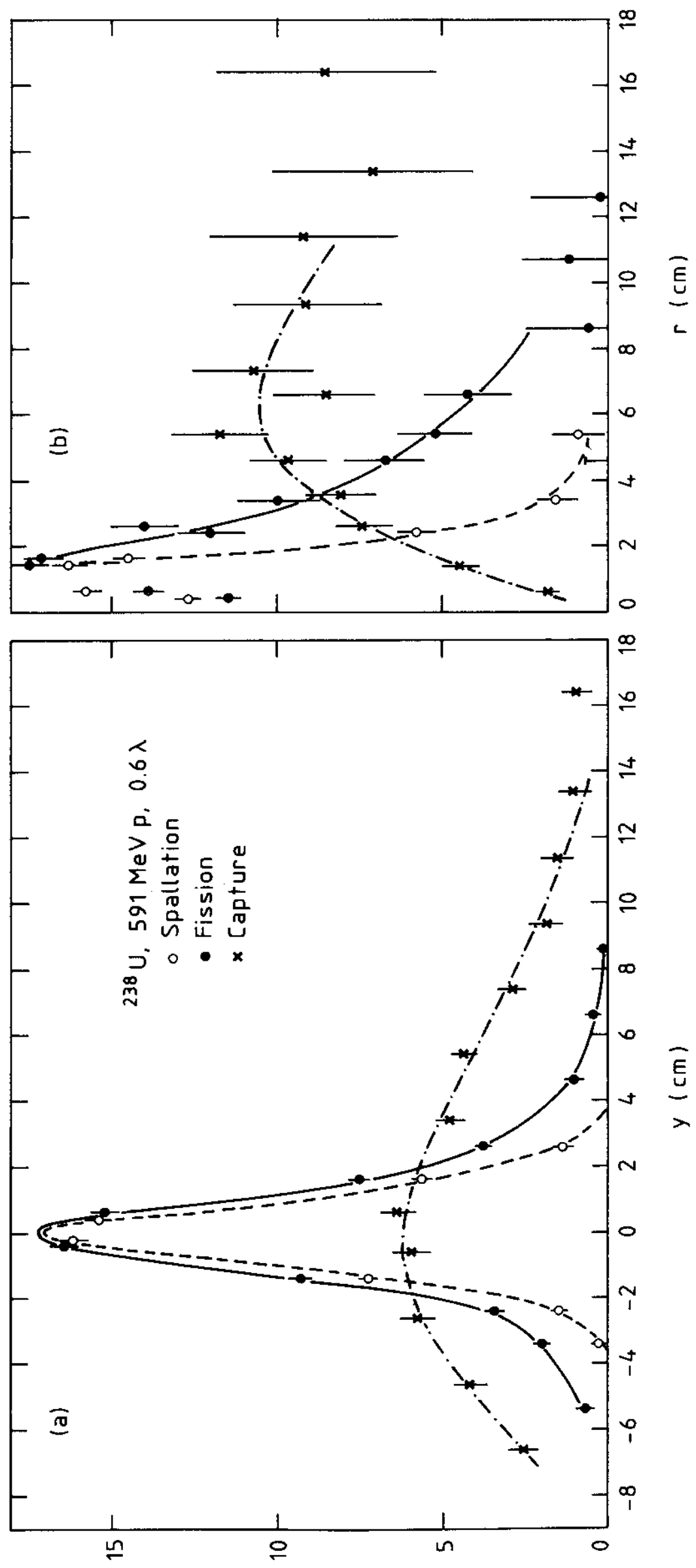

(st!unque) $z^{\text {mu }} /$ ! ! 


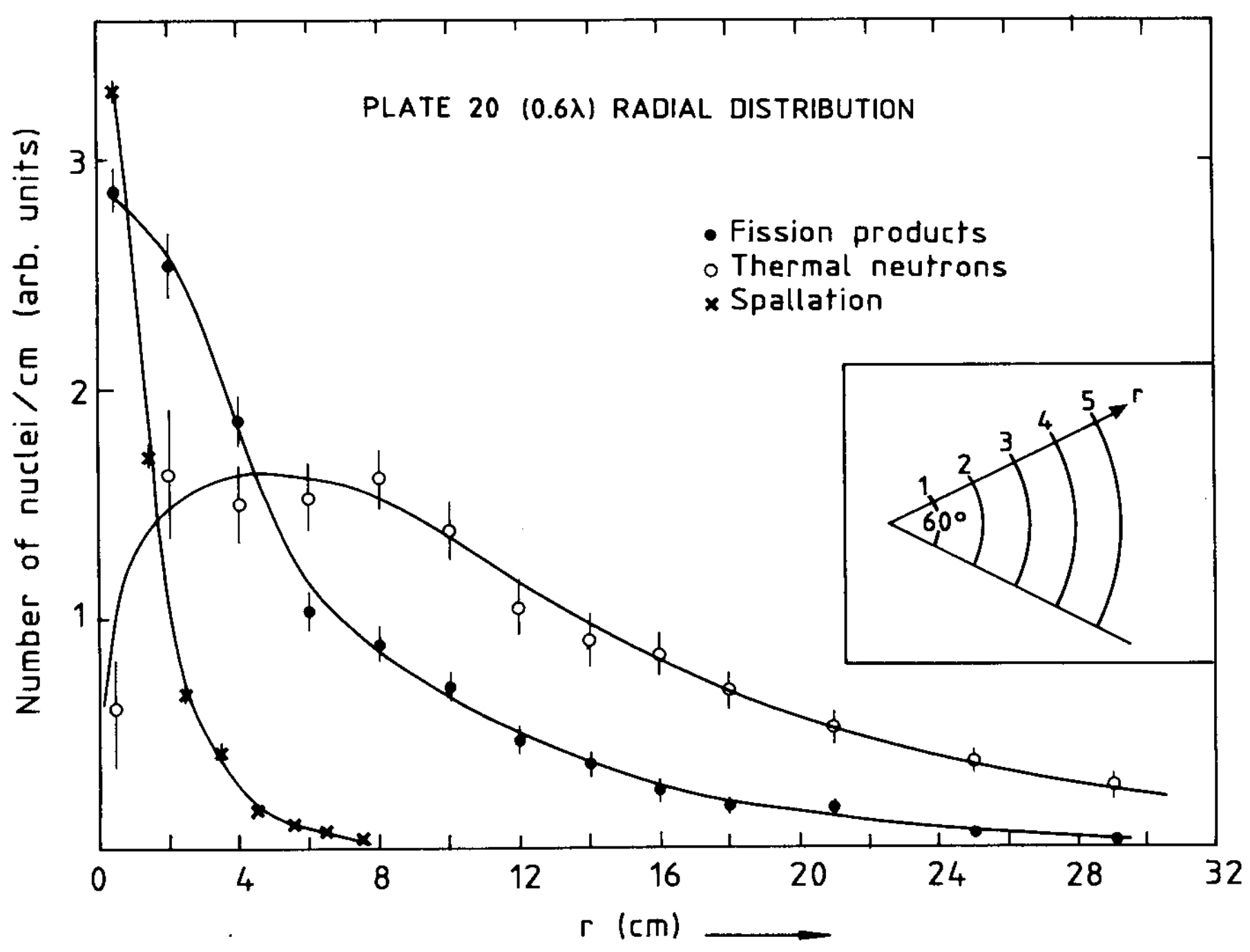

Fig. 7 


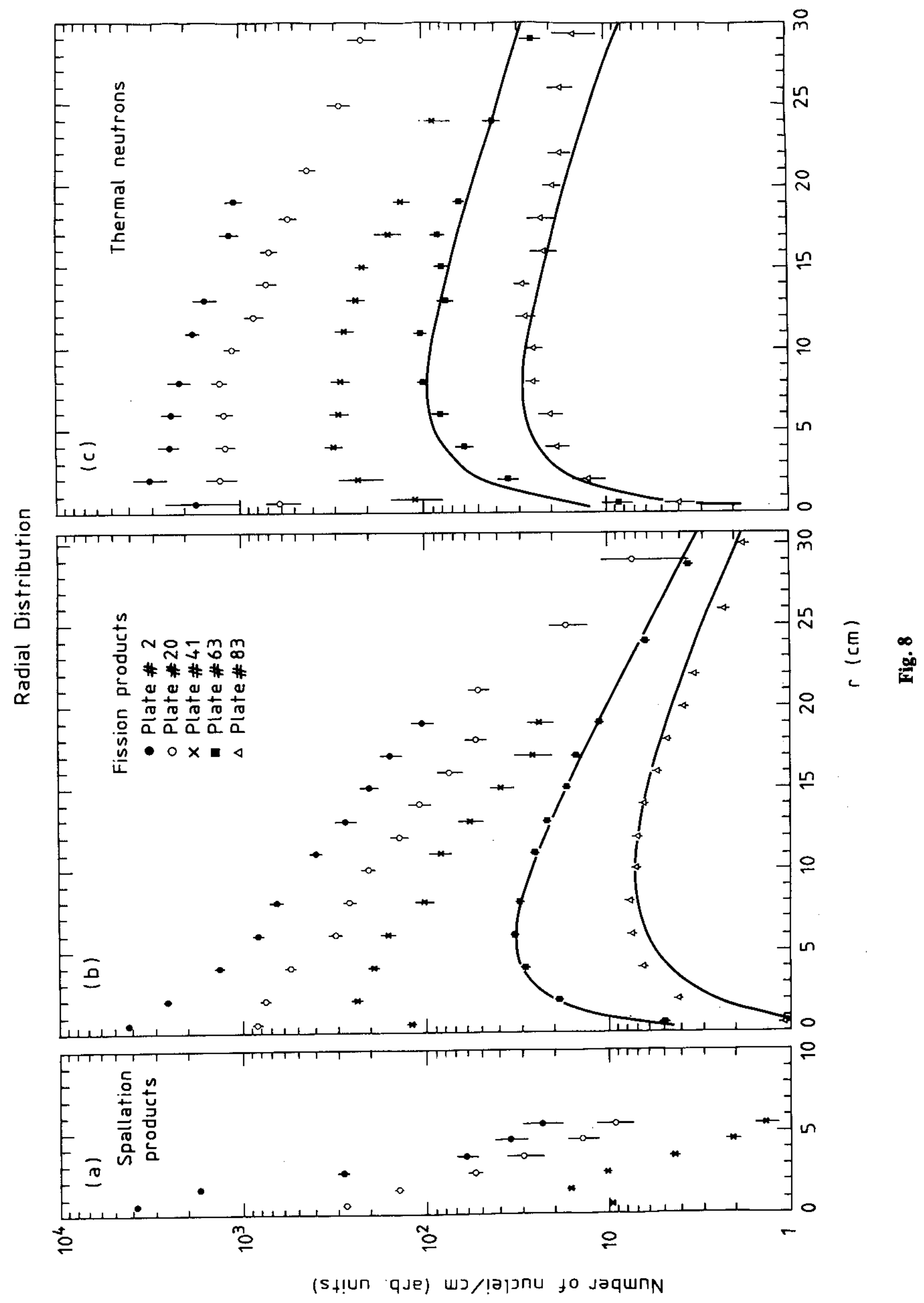




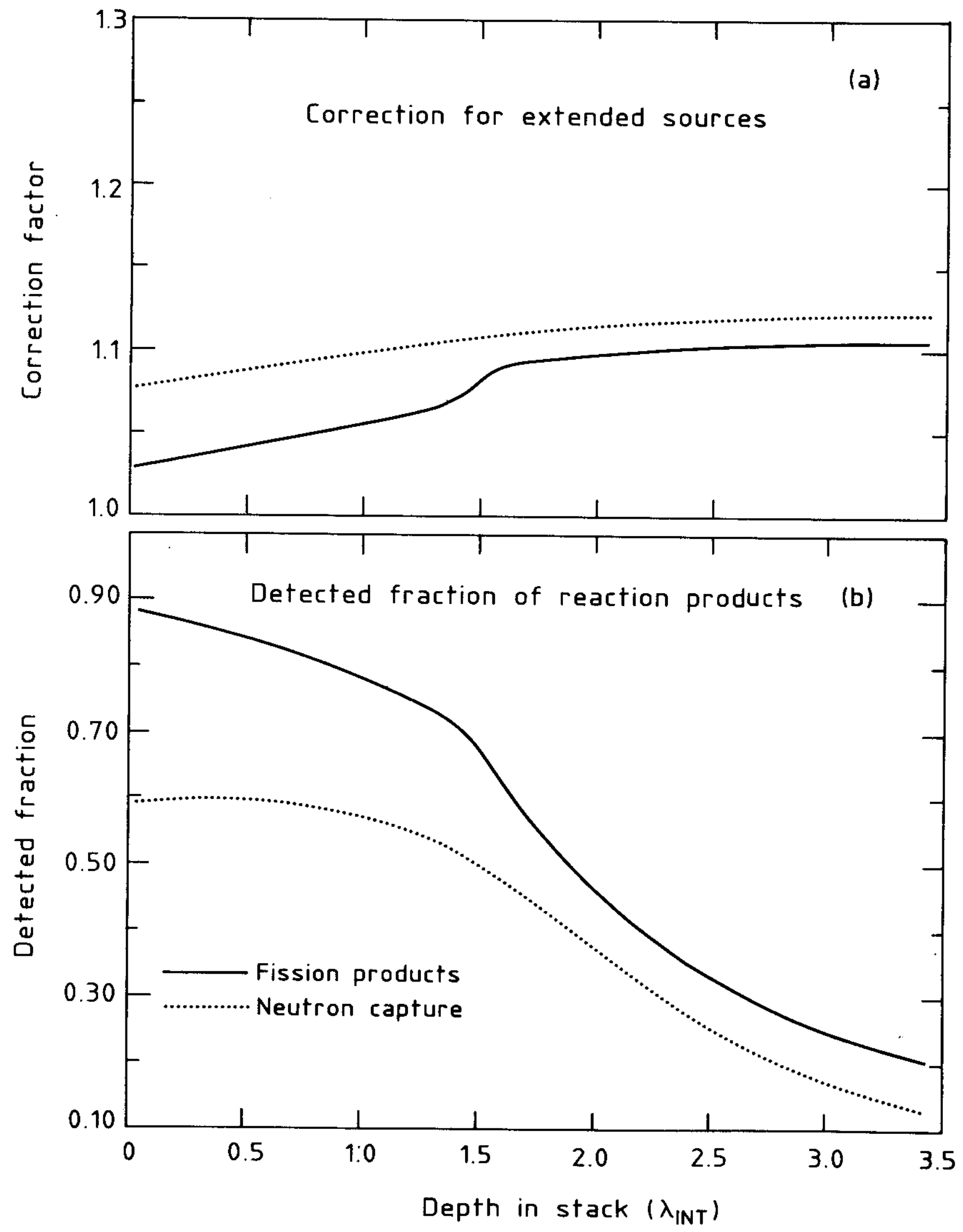

Fig. 9 


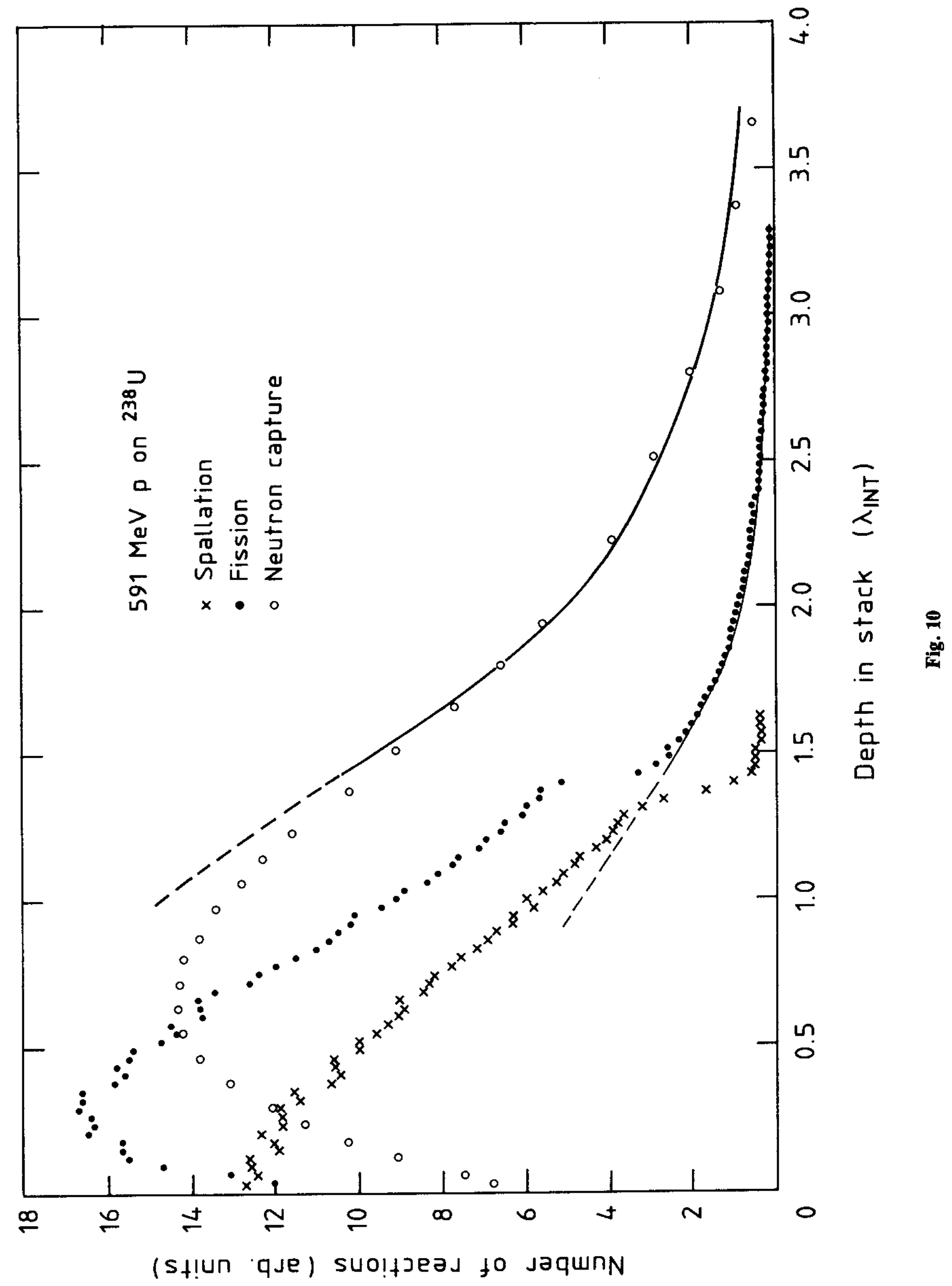




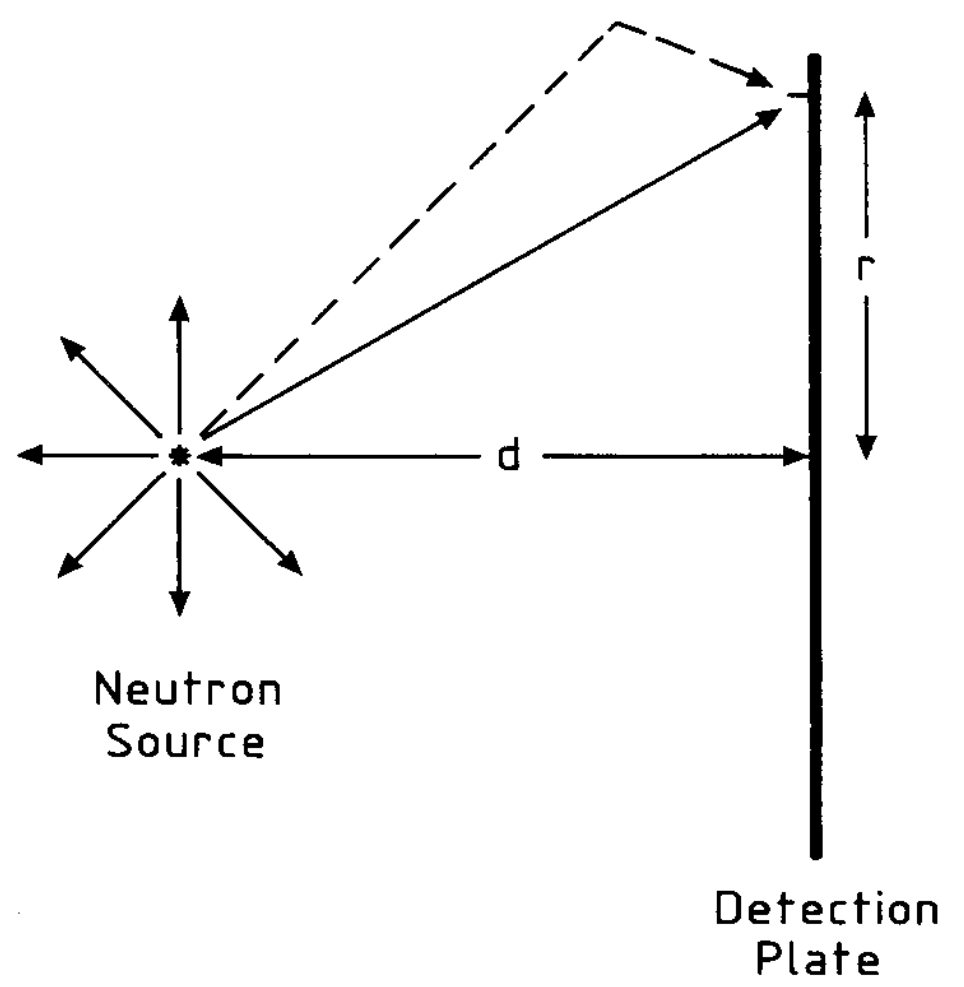

Fig. 11 


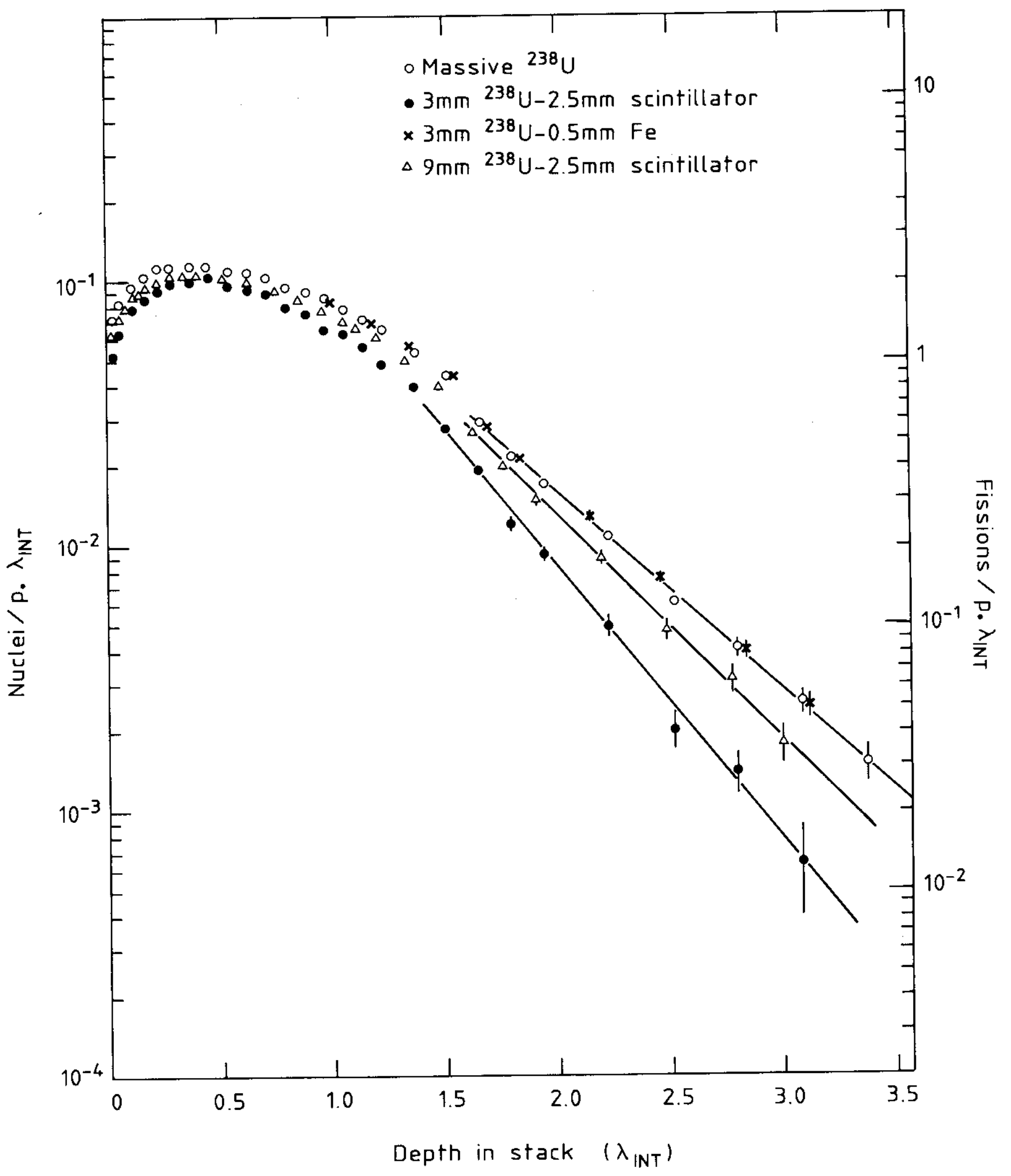

Fig. 12 


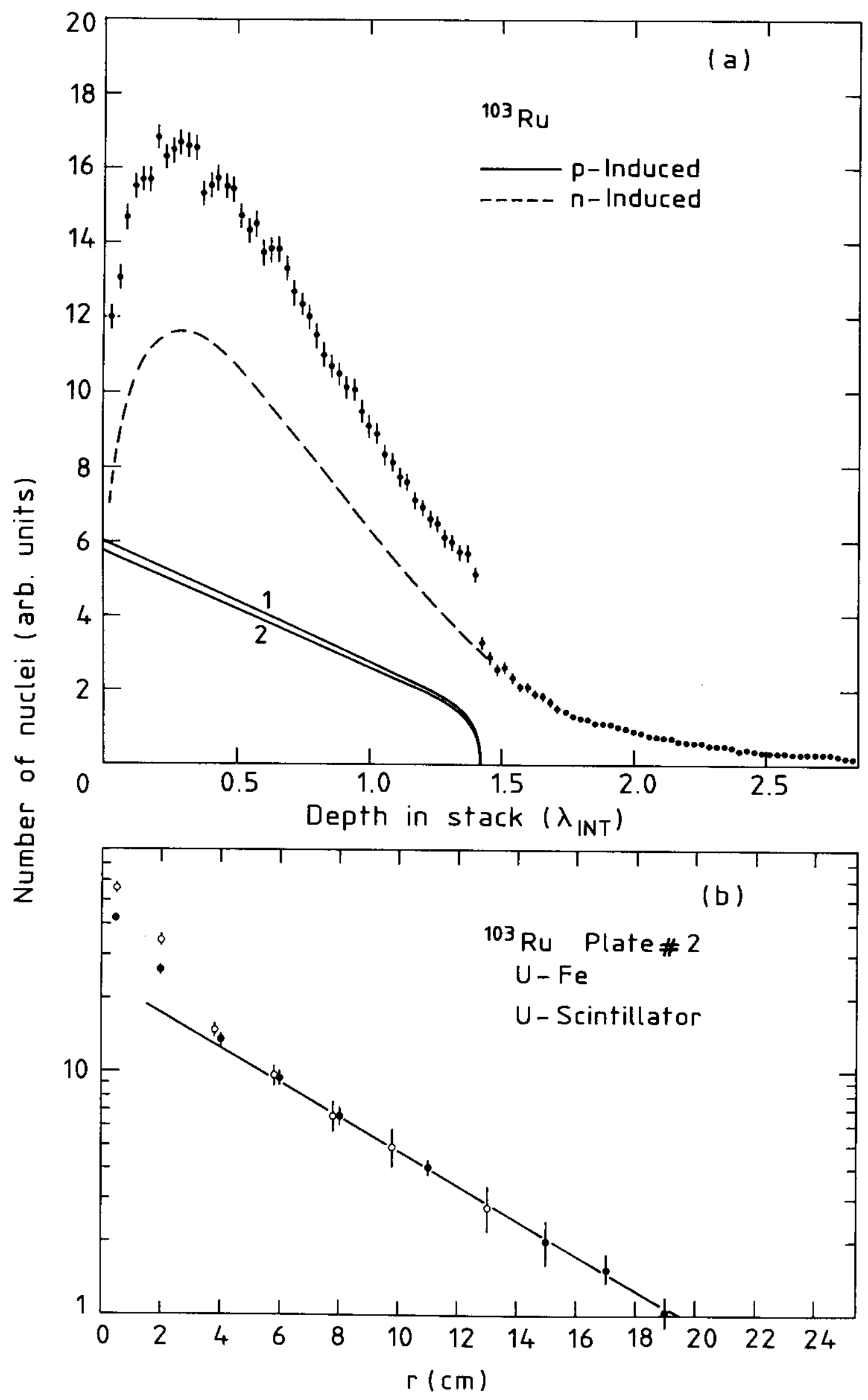

Fig. 13 


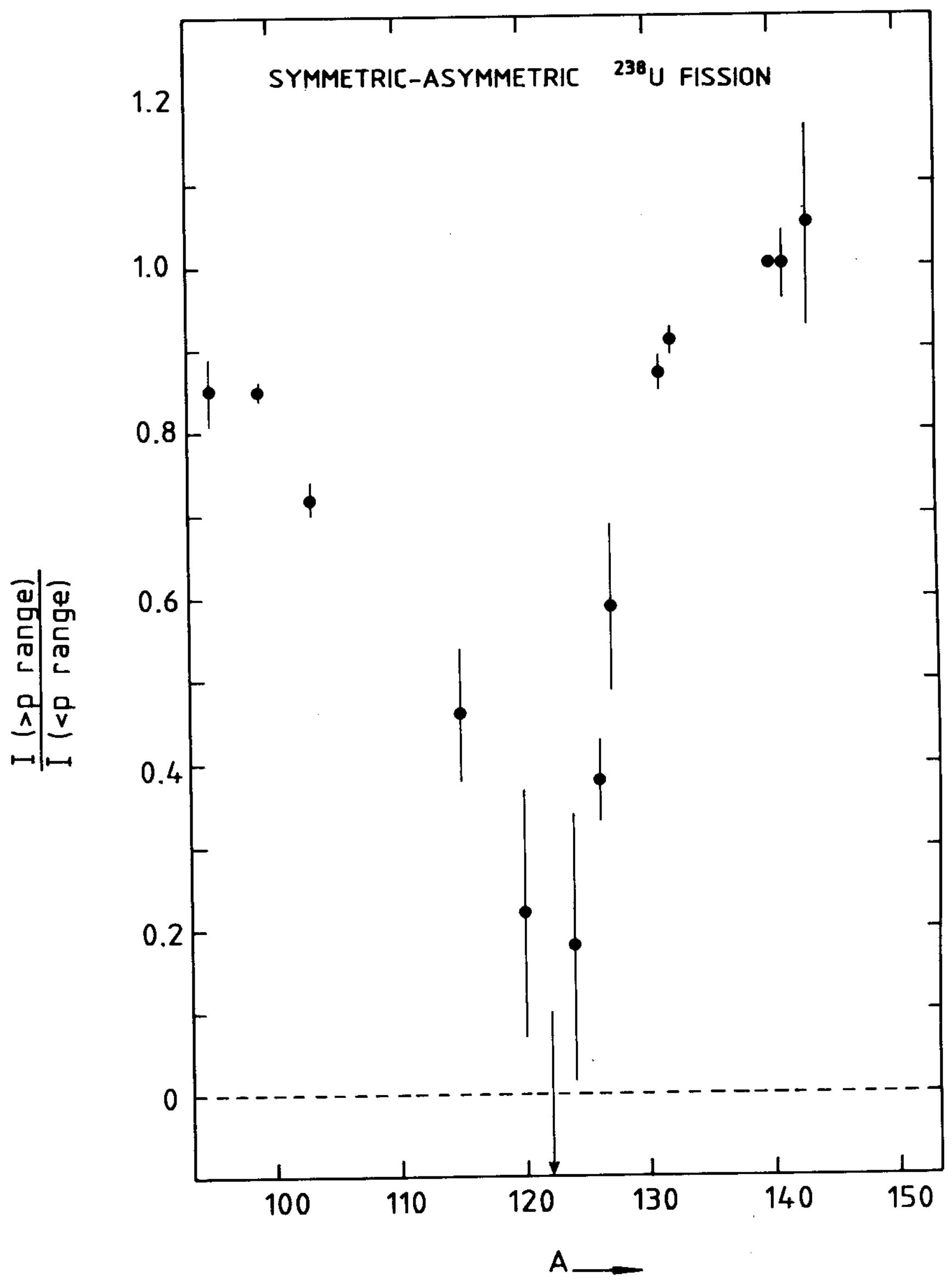

Fig. 14 

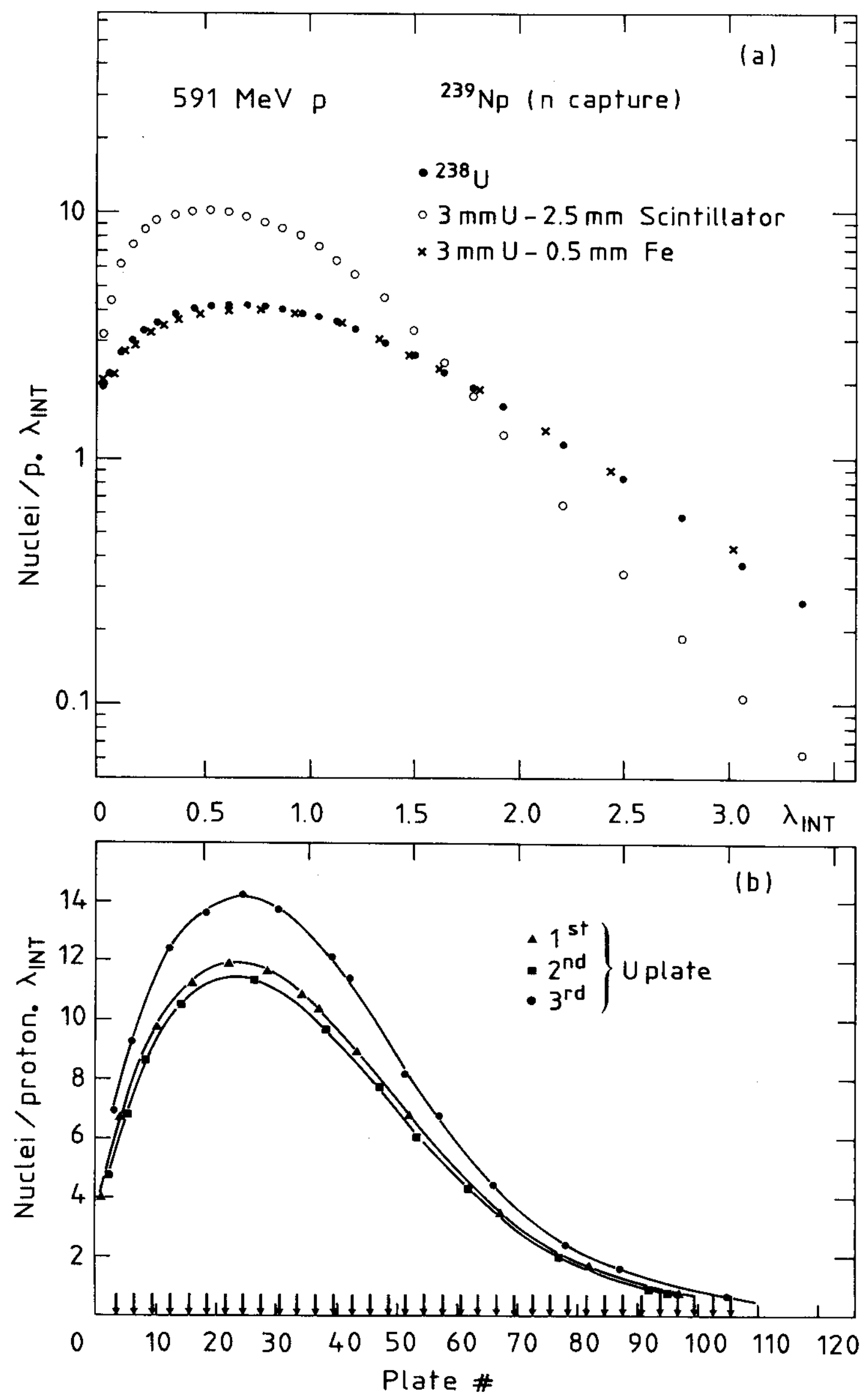

Fig. 15 


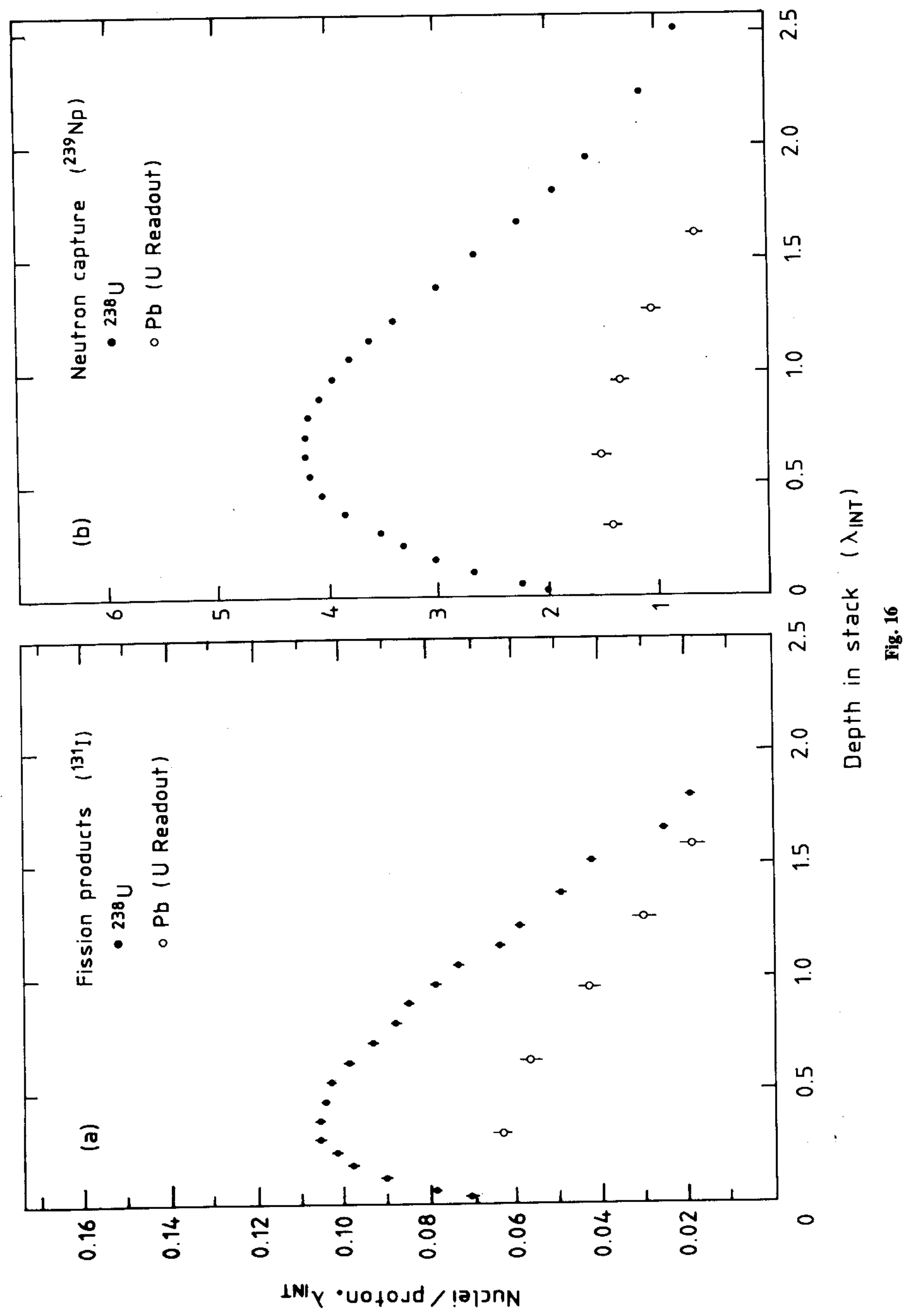


Fissions/ $\pi . \mathrm{cm}$

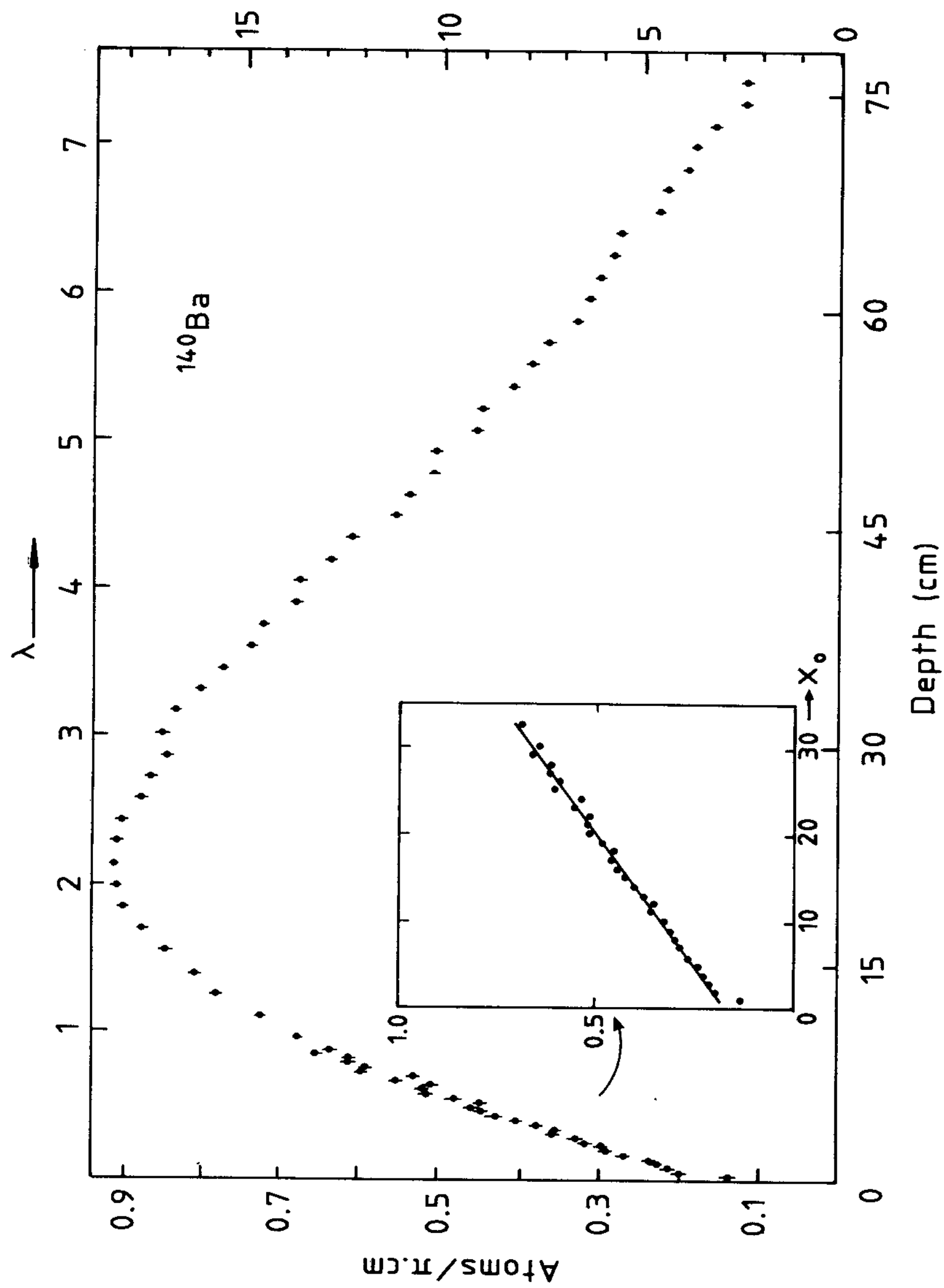




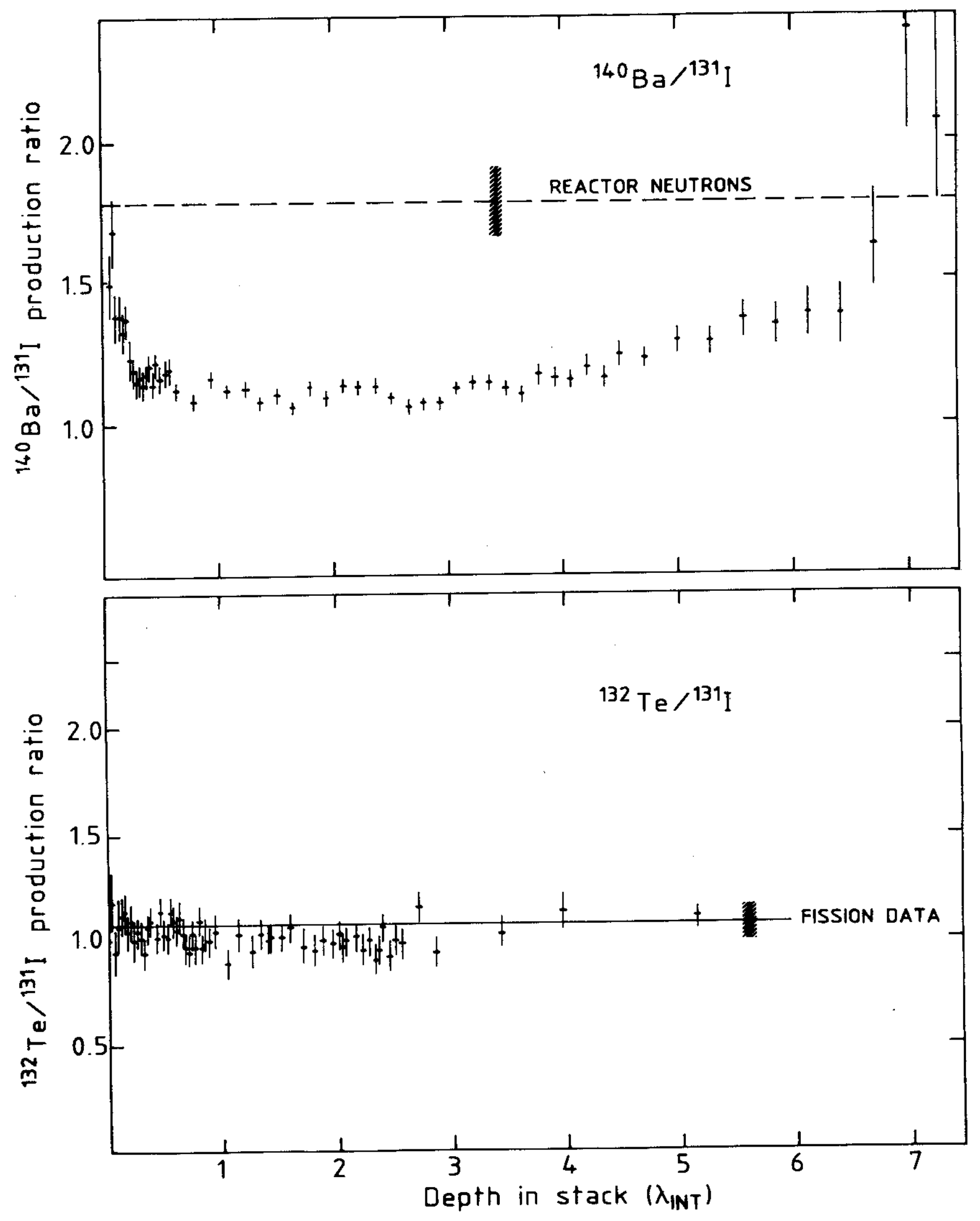

Fig. 18 


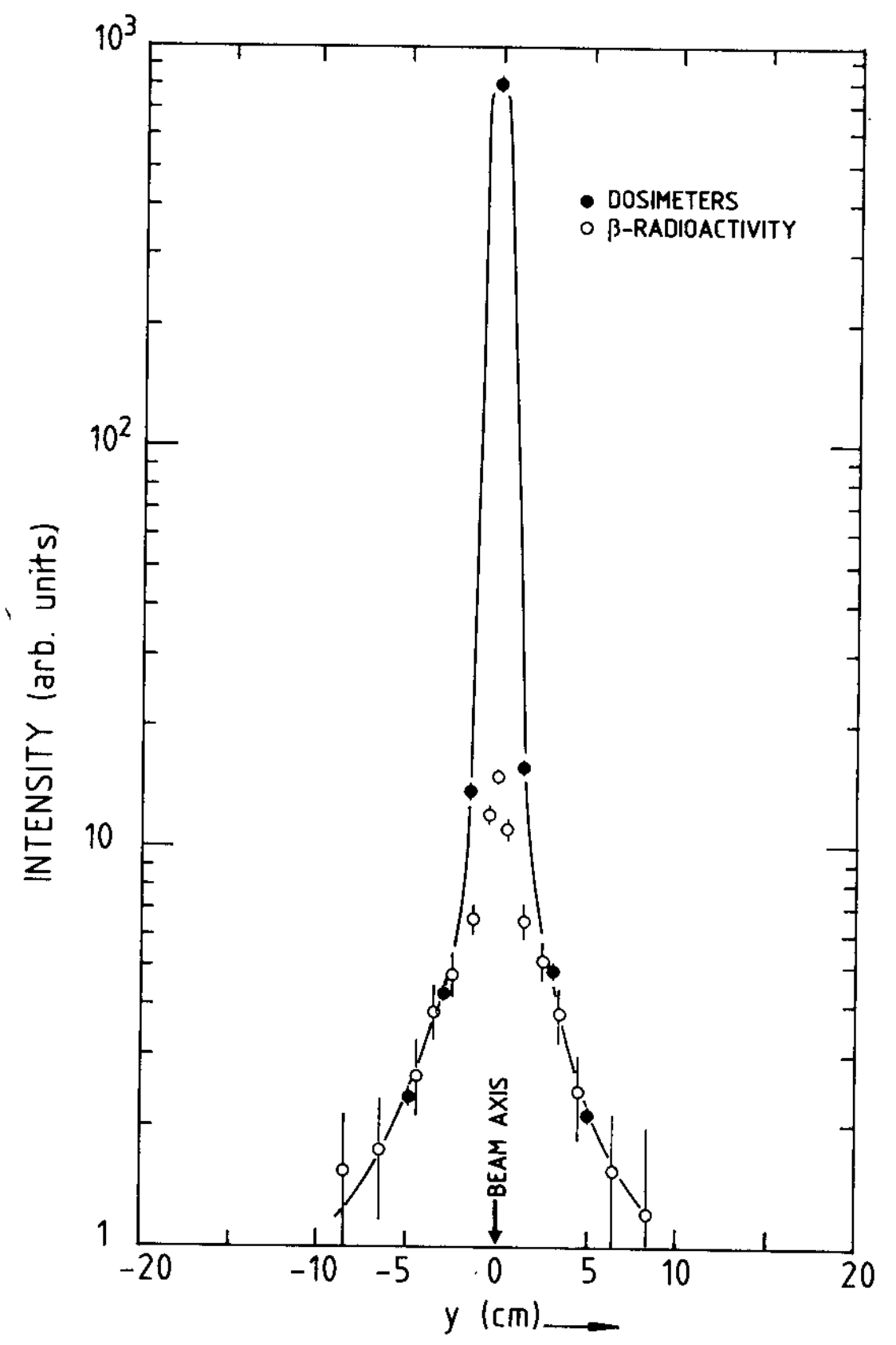

Fig. 19 


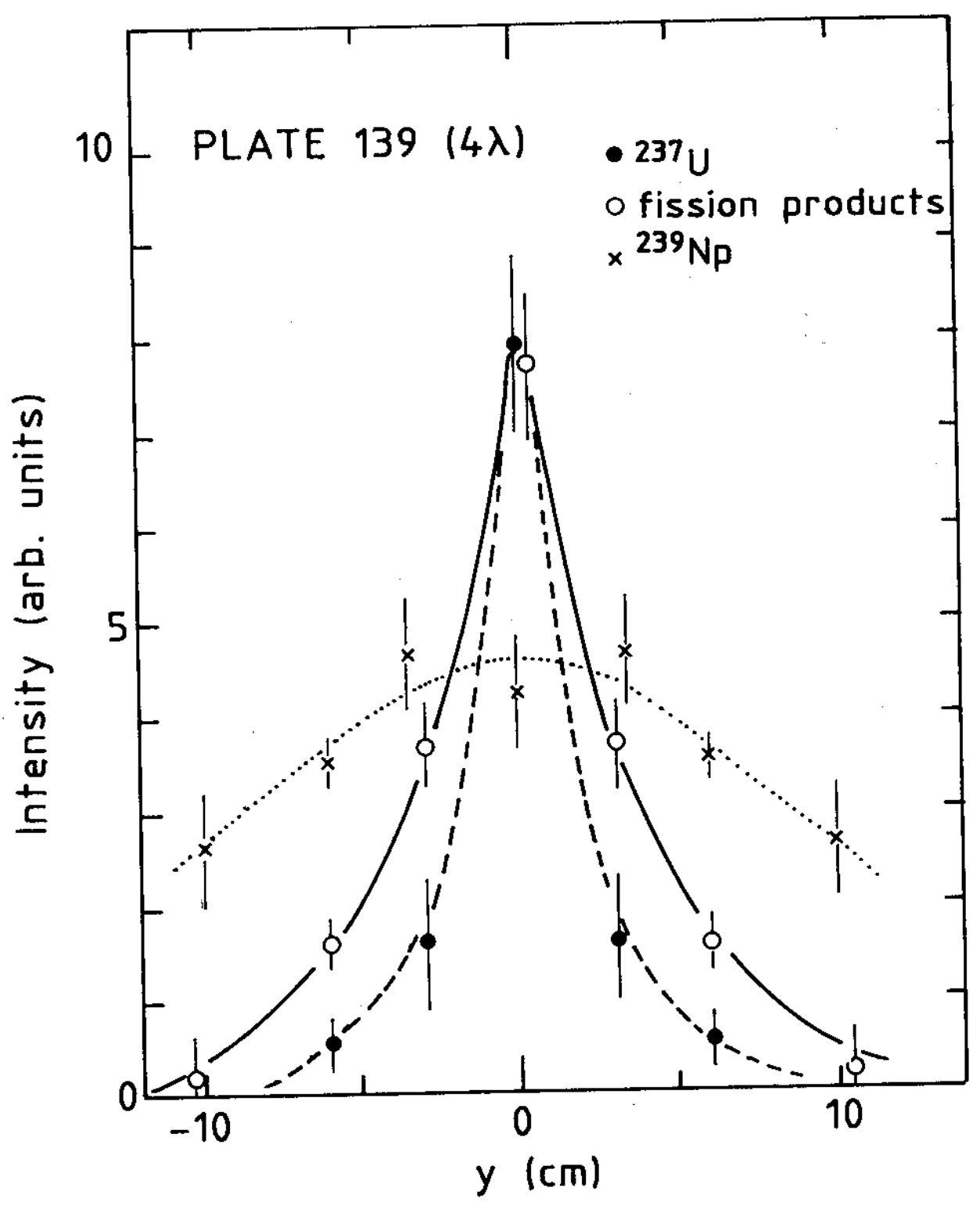

Fig. 20 

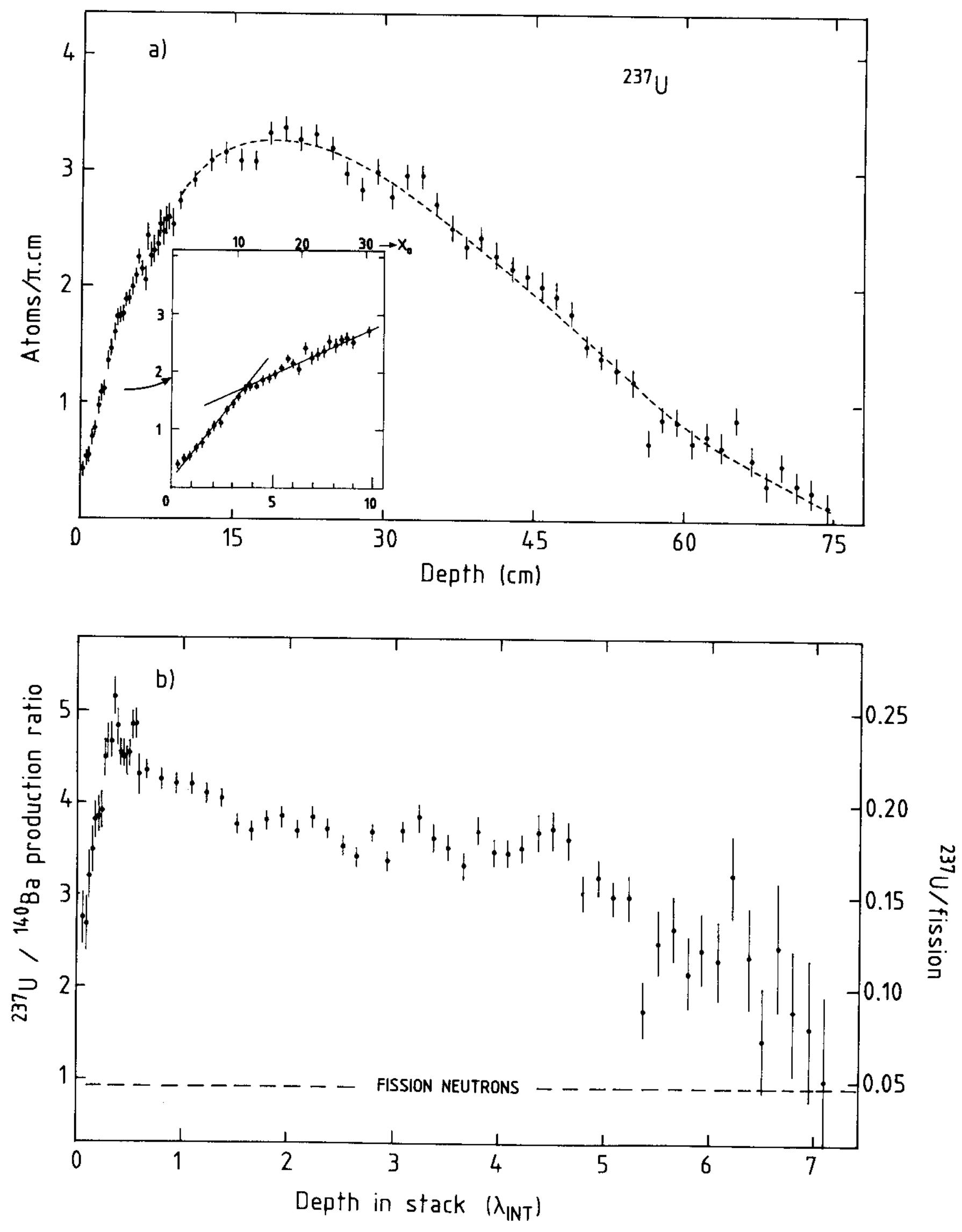

Fig. 21 

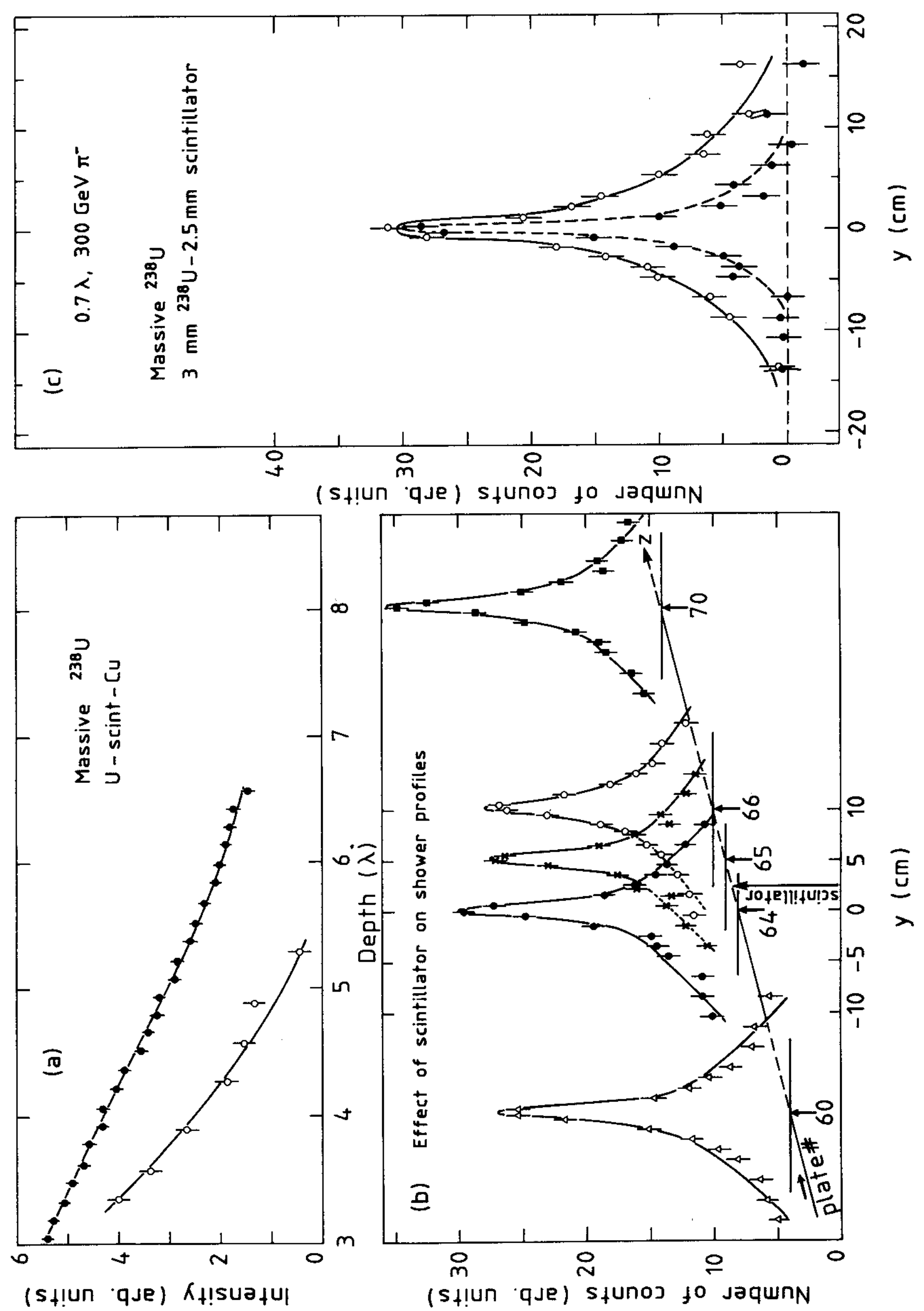

ป 


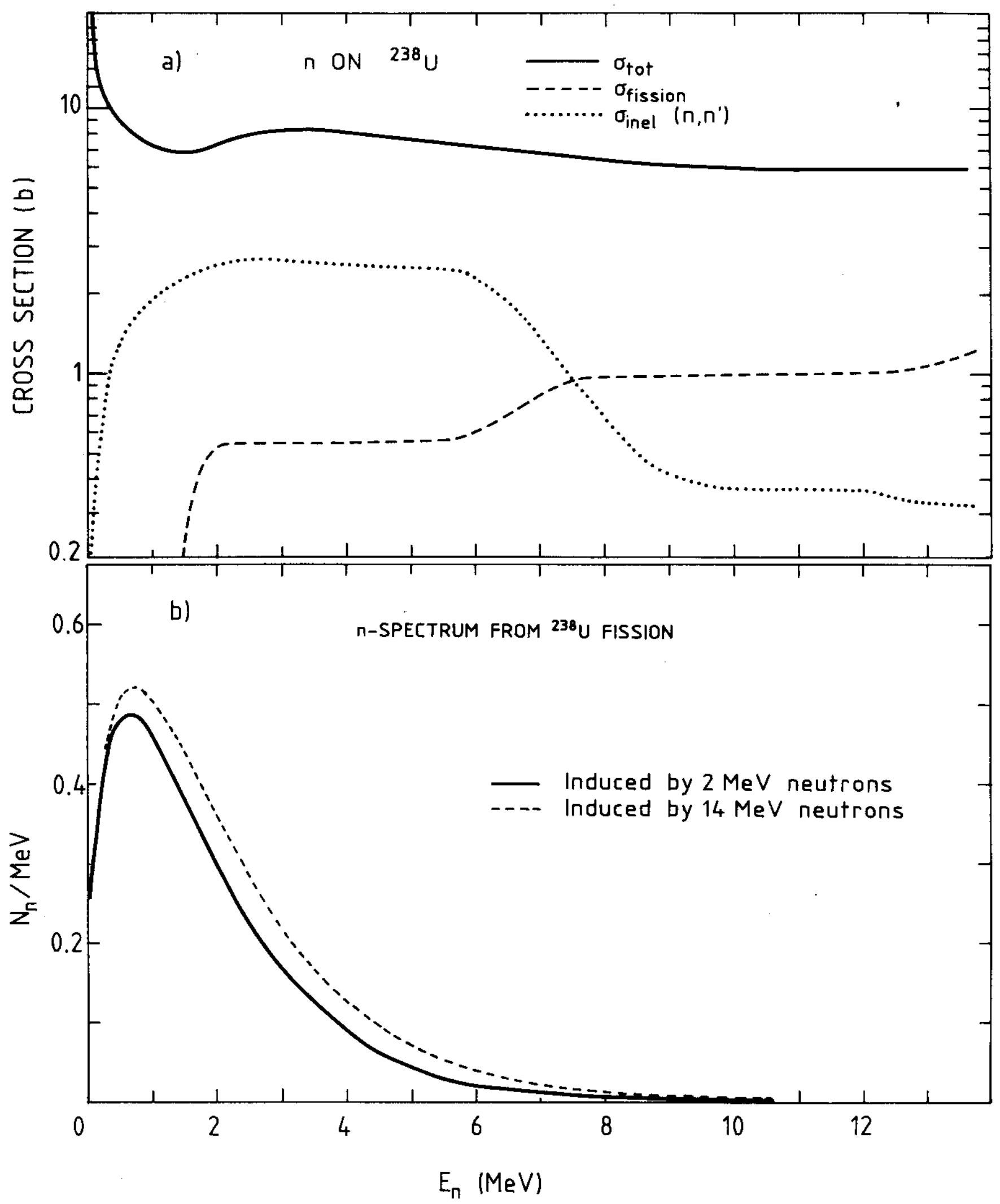

Fig. 23 


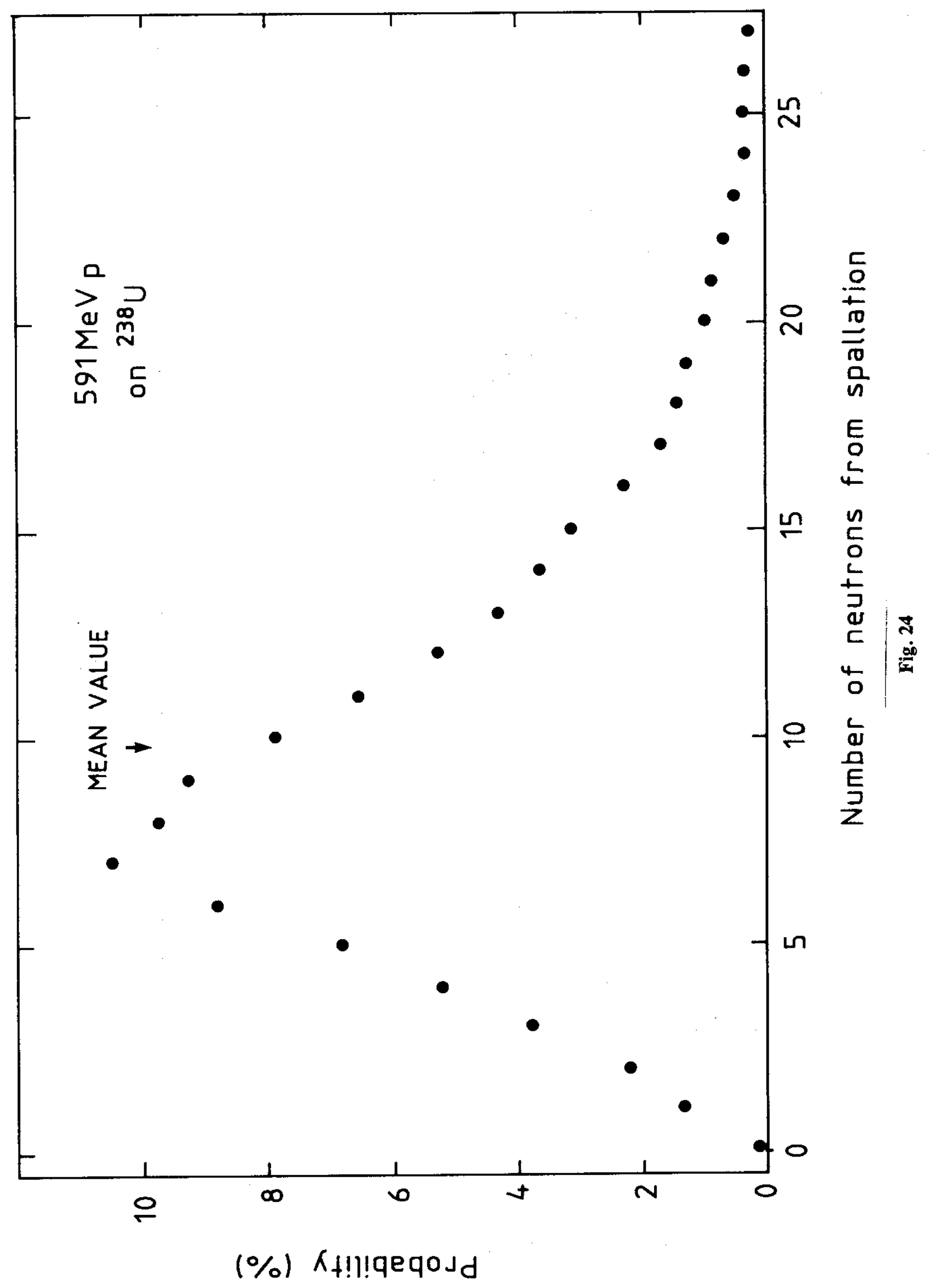




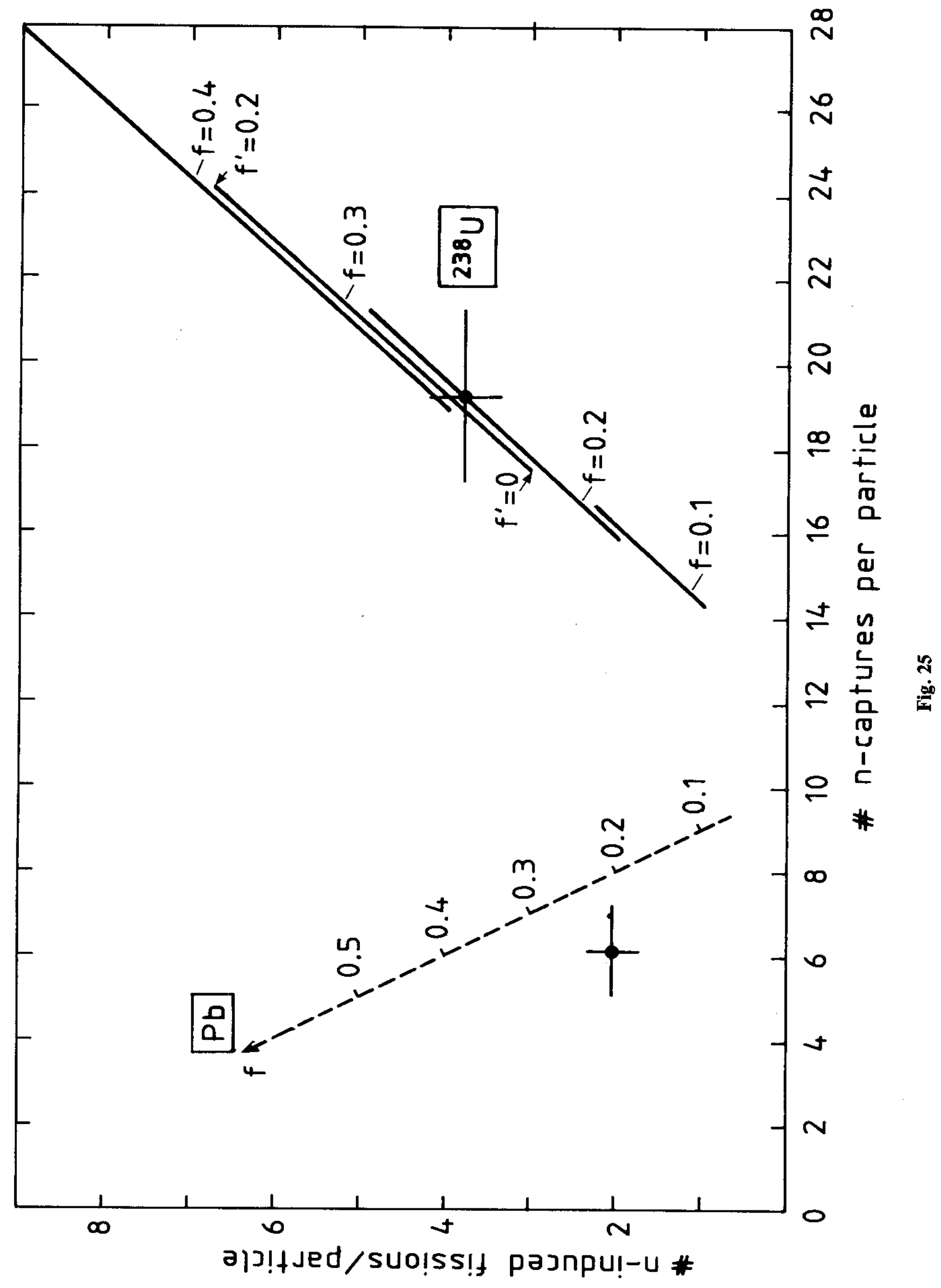




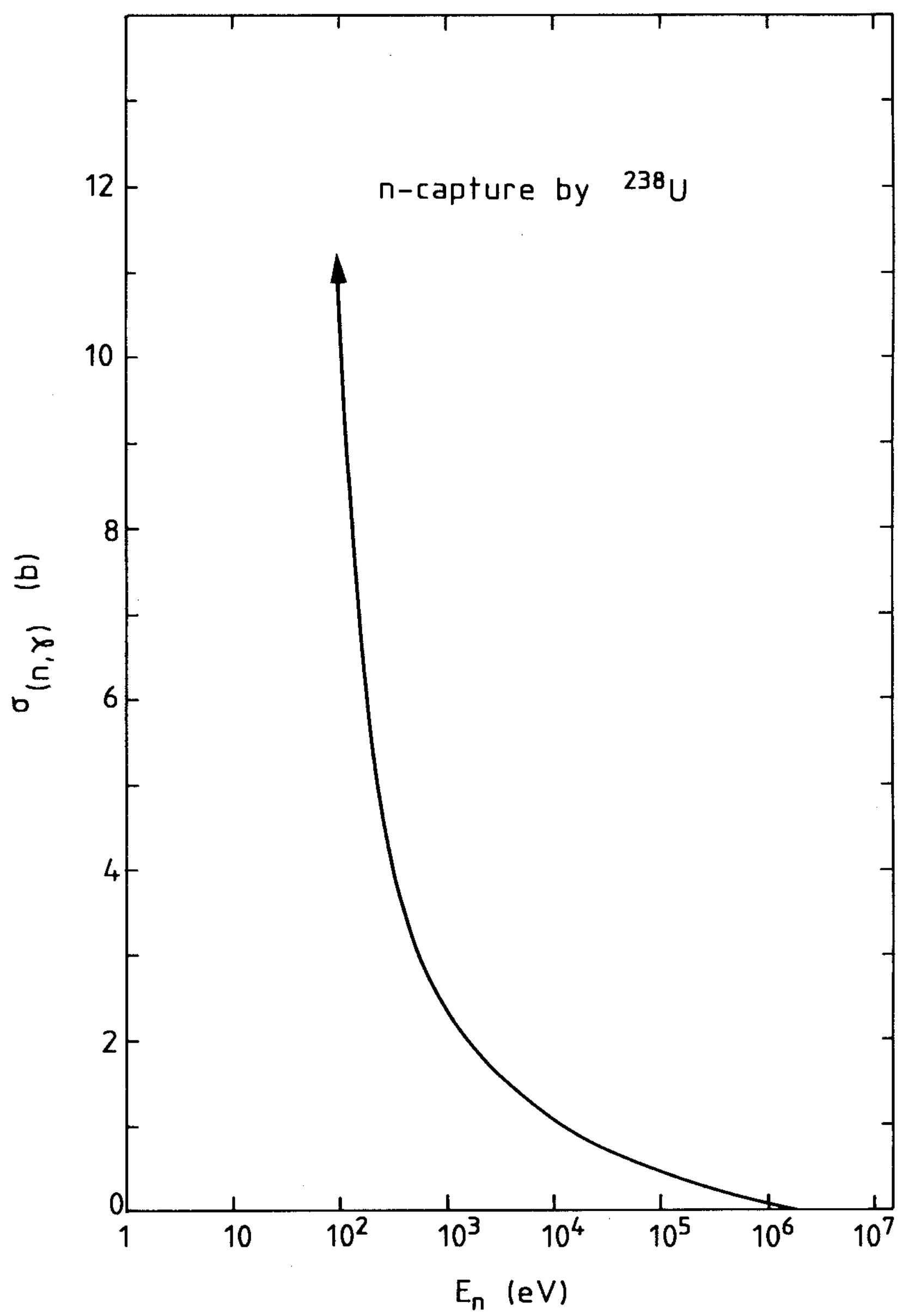

Fig. 26 